\title{
Geometric elliptic functionals and mean curvature
}

\author{
Luis J. Alías, Jorge H. S. DE LiRA AND MARCo Rigoli
}

\begin{abstract}
We introduce an extended notion of mean curvature for graphs via the Euler-Lagrange equation of a geometric elliptic functional. We then draw some geometric conclusions for Killing graphs with prescribed weighted and anisotropic mean curvatures with the aid of a general form of the weak maximum principle and a sufficient condition for an appropriate notion of parabolicity.
\end{abstract}

Mathematics Subject Classification (2010): 49Q20 (primary); 53C21, 53C42 (secondary).

\section{Introduction}

In this paper we introduce a generalized notion of mean curvature for hypersurfaces in Riemannian manifolds endowed with a Killing vector field. More precisely, the Riemannian manifolds we consider are warped products of the form $M \times_{\varrho} \mathbb{R}$, where $\varrho$ is the norm of a Killing vector field and $M$ is a complete Riemannian manifold. This generalized mean curvature characterizes critical hypersurfaces $\Sigma \subset M \times{ }_{\varrho} \mathbb{R}$ of geometric functionals of the form

$$
\int_{\Sigma} F e^{-f} d \Sigma
$$

where the Lagrangian $F$ is integrated against some weighted measure $e^{-f} d \Sigma$ on $\Sigma$. We assume that the Lagrangian is conserved by the flow generated by the Killing

This work was partially supported by MINECO/FEDER project MTM2012-34037 and Fundación Séneca project 04540/GERM/06, Spain. This research is a result of the activity developed within the framework of the Programme in Support of Excellence Groups of the Región de Murcia, Spain, by Fundación Séneca, Regional Agency for Science and Technology (Regional Plan for Science and Technology 2007-2010).

J. Lira was partially supported by CNPq/FUNCAP/PRONEX research project "Núcleo de Análise Geométrica e Aplicações" Grant PR2-0054-00009.01.00-11 .

M. Rigoli was partially supported by MEC Grant SAB2010-0073 and Fundación Séneca Grant 18883/IV/13, Programa Jiménez de la Espada.

Received July 30, 2013; accepted in revised form July 9, 2014.

Published online February 2016. 
field $Y$ in the sense that $\left.£_{Y} F\right|_{\Sigma}=0$. This last assumption guarantees that, if the critical hypersurface is a graph over some domain in $M$, the Euler-Lagrange equation for the functional (1.1) can be written in terms of a partial differential equation on $M$. For details, see Theorem 2.1 in Section 2 below.

As expected, when considering the Riemannian product $M \times \mathbb{R}$, an appropriate choice of the Lagrangian and of the measure yields the usual definition of the mean curvature of the graph $\Sigma$ of a function $u \in \mathcal{C}^{\infty}(M)$ via the well-known mean curvature equation

$$
\operatorname{div}\left(\frac{\nabla u}{\sqrt{1+|\nabla u|^{2}}}\right)=n H,
$$

where $n=\operatorname{dim} M$ and $H$ is the standard mean curvature of the graph in the direction of the normal

$$
N=\frac{1}{\sqrt{1+|\nabla u|^{2}}}\left(-\nabla u+\frac{\partial}{\partial s}\right),
$$

where $s$ is the coordinate on $\mathbb{R}$. Here the operators div and $\nabla$ are defined on $M$.

One of the main goals of this paper is to elucidate the interplay between constant or assigned mean curvature graphs, in the aforementioned warped products, and the analysis of weighted elliptic operators defined on the smooth metric measure space $\left(M, e^{-f} d M\right)$ where the weight is appropriately related to the warping function. The interplay comes from the following remark. Let the weighted divergence operator be

$$
\operatorname{div}_{f} X=e^{f} \operatorname{div}\left(e^{-f} X\right), \quad X \in \Gamma(T M),
$$

that naturally appears in connection with the weighted volume form $e^{-f} d M$ on $M$. It turns out that solutions of the weighted mean curvature equation

$$
\operatorname{div}_{f}\left(\frac{\nabla u}{\sqrt{1+|\nabla u|^{2}}}\right)=n H_{f},
$$

define, at the same time, graphs with prescribed weighted mean curvature in $M \times \mathbb{R}$ and graphs with prescribed mean curvature in warped products $M \times{ }_{\varrho} \mathbb{R}$ provided that $f=-\log \varrho$.

One of the main issues in this context is the existence and uniqueness of entire graphs with constant mean curvature in $M \times_{\varrho} \mathbb{R}$, where $M$ is non-compact. Quite recently, H. Rosenberg, F. Schulze and J. Spruck [26] proved that if $M$ has non-negative Ricci curvature and sectional curvatures bounded from below, then an entire minimal graph lying into a half-space in the Riemannian product $M \times \mathbb{R}$ is a slice of the form $M \times\{c\}$ for some $c \in \mathbb{R}$. The same conclusion holds for entire graphs with constant mean curvature lying into slabs of a warped product $M \times_{\varrho} \mathbb{R}$ if one adds the assumption that the warping factor is uniformly bounded up to its second derivatives, [12]. Both results are based on Liouville-type properties for the 
mean curvature equation and rely on refined gradient estimates essentially due to N. Korevaar [21]. In [12] a version of the maximum principle [23] is also used for guaranteeing that, under those assumptions, entire graphs with constant mean curvature are indeed minimal.

In this paper we derive a maximum principle for a wide class of quasilinear elliptic operators that includes the generalized mean curvature operator. This allows us to prove a number of non-existence and uniqueness results for entire graphs with either assigned or constant generalized mean curvature in warped product spaces. An interesting feature of these results is that the influence of the warping factor dictates what should be the regions where the maximum principle holds. In rough terms, these regions are bounded by the graphs of powers of the distance from a fixed origin of $M$. This specific power is, in turn, related to the growth rate of the mean curvature. Thus these radial functions can be understood as barriers bounding the regions that play the role of slabs or half-spaces. Moreover, these functions yield global quantitative height estimates for entire graphs.

In the statement of the results, $B_{R}$ denotes the geodesic ball in $M$ with radius $R$ centered at some point $o \in M$ and $r(x)=\operatorname{dist}_{M}(o, x), x \in M$. The following is a corollary of Theorem 4.1 in Section 4:

Given a positive function $\varrho \in \mathcal{C}^{\infty}(M)$ consider the warped product manifold $\bar{M}=M \times \varrho \mathbb{R}$. Assume that

$$
\sup _{M} \varrho<+\infty
$$

and

$$
\liminf _{R \rightarrow+\infty} \frac{\log \int_{B_{R}} \varrho}{R^{2-\varsigma}}=0
$$

for some $0 \leq \varsigma<2$. Then any constant mean curvature graph $\Sigma$ in $\bar{M}$ lying between the graphs of the radial functions $\pm \beta r^{\varsigma}$ outside a compact in $M$, for some $\beta>0$, is minimal. In particular, if $\varsigma=0$, the sectional curvatures of $M$ satisfy $K_{M} \geq-K_{0}$ for some positive constant $K_{0}$ and $\operatorname{Ric}_{M} \geq 0$, then $\Sigma=M \times\{c\}$ for some $c \in \mathbb{R}$.

The precise notion of graphs into warped products is formalized in terms of Killing graphs in Section 2. In Section 3, we prove the maximum principle we alluded to, following techniques introduced in our previous works [1,24,25]. This new form of a general weak maximum principle, stated as Theorem 3.2 in Section 3, holds for possibly unbounded functions and extended divergence-type operators. As we mentioned above, these operators include those coming from the Euler-Lagrange equations of the elliptic functionals we are considering in Section 2. In the same spirit we have also given a sufficient condition for a related notion of parabolicity as expressed in Theorem 3.4 in Section 3. Since these results are interesting in their own and could be used in different contexts, we have given self-contained proofs. 
This enlarged notion of parabolicity adapted to the generalized mean curvature operator bears fruitful consequences like the next result, that is a particular case of Theorem 4.6 in Section 4:

Let $M, \varrho$ and $\bar{M}$ be as above. Suppose that

$$
\sup _{M} \varrho<+\infty
$$

and

$$
\frac{1}{\int_{B_{R}} \varrho} \notin L^{1}(+\infty) .
$$

Then any constant mean curvature graph $\Sigma$ lying into a slab of the form $M \times\left[s_{1}, s_{2}\right]$ with $s_{1}<s_{2}$ is of the form $M \times\{c\}$ for some $c \in\left[s_{1}, s_{2}\right]$.

Another consequence of the parabolicity is the following (see Theorem 4.13 in Section 4 for a more general statement):

Let $\Sigma$ be the graph of a function $u$ in $M \times_{\varrho} \mathbb{R}$. Suppose that

$$
\varrho^{2} \in L^{1}(M, d M)
$$

and that $|\nabla u|=O\left(\varrho^{-1}\right)$ as $r \rightarrow+\infty$. If the mean curvature vector of the graph points in the same direction of the positive orientation of the factor $\mathbb{R}$ and $u$ is bounded from above, then there exists $c \in \mathbb{R}$ such that $\Sigma=M \times\{c\}$.

The geometric applications of the analytical theorems in Section 3 are gathered together in Sections 4 and 5 where we specialize our approach to the cases of the classical, weighted and anisotropic notions of mean curvature.

Killing graphs with constant anisotropic mean curvature are interesting examples of extremal hypersurfaces for certain choices of the functional in (1.1). In Euclidean space the compact minimizers of these functionals, known as Wulff shapes, have been studied by Jean Taylor [29] who gave a rigorous existence proof using Geometric Measure Theory to deal with the so-called crystalline functionals, which is singular; this type of functionals is not covered in the present paper. There is a further approach to the problem called the level set approach especially used for the anisotropic curvature flow [15]. Finally, the anisotropic volume preserving mean curvature flow has also been studied in the case of vector spaces with a Minkowski norm by B. Andrews [2].

In spite of a massive list of distinguished contributions, the differential geometric aspects of the anisotropic mean curvature have still to be unfolded in depth. To the best of our knowledge some of the main contributions on the subject are, among others, due to T. Colding and W. Minicozzi [9], M. Koiso and B. Palmer [19, 20], and Y. He and H. Li [16]. This line of research is concerned, for instance, with the stability of the Wulff shape as well as with the study of critical compact hypersurfaces for higher order anisotropic mean curvatures. Furthermore, Koiso and Palmer 
wrote a beautiful series of papers on capillary problems involving elliptic parametric functionals associated to the same type of Lagrangian we define in equation (5.25) below.

Another important trend has been developed by H. von der Mosel, U. Clarenz, S. Hildebrandt and others $[7,8,17,18]$. They emphasize the analytical side, for instance, the existence and regularity of solutions of the Plateau problem and so on. In [22], we combine both approaches to formulate the notion of anisotropic mean curvature in general Riemannian manifolds. There we prove that, if the ambient Riemannian space is endowed with a Killing field, then it is possible to guarantee the existence of critical points for Lagrangians conserved by the field flow. This is the starting point of the present research.

ACKNOWLEDGEMENTS. The authors would like to thank the anonymous referee for his/her valuable suggestions and corrections which contributed to improve this paper. This work was started while the first and third authors were visiting the Departamento de Matemática of the Universidade Federal do Ceará, Fortaleza, Brazil, and it also benefited from two visits of the second and third authors to the Departamento de Matemáticas of the Universidad de Murcia, Spain. The authors would like to thank both institutions for their hospitality.

\section{A variational setting for a generalized mean curvature}

Let $\bar{M}$ be an $(n+1)$-dimensional Riemannian manifold with metric and Riemannian connection respectively denoted by $\bar{g}=\langle\cdot, \cdot\rangle$ and $\bar{\nabla}$. The norm derived from $\bar{g}$ is denoted by $|\cdot|$. In what follows we fix a differentiable positive function $F: T \bar{M} \backslash\{0\} \rightarrow \mathbb{R}$ satisfying the homogeneity condition

$$
F(y, t \eta)=t F(y, \eta),
$$

for all $(y, \eta) \in T \bar{M}, \quad \eta \neq 0, t>0$. According to [8], we refer to $F$ as to a parametric Lagrangian. The parametric Lagrangian $F$ allows us to introduce a parametric functional $\mathcal{F}$ as follows. Given an oriented $n$-dimensional Riemannian manifold $\Omega$ and $\psi: \Omega \rightarrow \bar{M}$ an immersion, we define

$$
\mathcal{F}[\psi]=\int_{\Omega} F(\psi, N) \psi^{*} d \bar{M},
$$

where the volume element $\psi^{*} d \bar{M}$ is induced by the immersion $\psi$ and $N$ is a unit normal vector field along $\psi$. The classical example of such a functional is the volume of the immersion, which corresponds to the Lagrangian $F(y, \eta)=|\eta|$. Another important class of examples are provided by functionals of the form

$$
\mathcal{F}_{f}[\psi]=\int_{\Omega} e^{-f} F(\psi, N) \psi^{*} d \bar{M},
$$


for some differentiable function $f \in \mathcal{C}^{\infty}(\Omega)$. We briefly comment on the ellipticity of the functional later in Section 3.

Given an arbitrary vector field $Q \in \Gamma(T \bar{M})$ an important example is the following generalized version of Hildebrandt's functional

$$
\mathcal{F}_{f}[\psi]+\int_{\Omega} e^{-f}\langle Q(\psi), N\rangle \psi^{*} d \bar{M} .
$$

Note that with the choices $f=0, F(y, \eta)=|\eta|$ and $Q(\psi)=\psi$ the critical points of this functional, in the case of $\bar{M}=\mathbb{R}^{n} \times \mathbb{R}$, model capillary surfaces. We refer the reader to [8] for further details.

Here we are mainly concerned with hypersurfaces described as graphs in warped product spaces. Specifically, given a complete Riemannian manifold $(M, \sigma)$ and a positive function $\varrho \in \mathcal{C}^{\infty}(M)$, we consider the warped product space $\bar{M}=$ $M \times \times_{\varrho} \mathbb{R}$, that is, the product manifold $M \times \mathbb{R}=\{(x, s): x \in M, s \in \mathbb{R}\}$ endowed with the Riemannian metric

$$
\bar{g}=\sigma+\varrho^{2}(x) d s^{2} .
$$

In this setting, the coordinate vector field $\frac{\partial}{\partial s}$ is a non-singular Killing vector field on $\bar{M}$. Given a smooth function $u$ defined on an open domain $\Omega \subset M$, the graph of $u$ is the hypersurface given by

$$
\Sigma(u)=\{(x, u(x)): x \in \Omega\} \subset M \times \mathbb{R} .
$$

More generally, assume that $\bar{M}$ is a Riemannian manifold endowed with a nonsingular Killing vector field $Y$ with complete flow lines such that the orthogonal distribution

$$
y \in \bar{M} \mapsto \mathcal{D}_{y}=\left\{v \in T_{y} \bar{M}:\langle Y(y), v\rangle=0\right\} \subset T_{y} \bar{M}
$$

is integrable. It is easy to verify that the integral leaves of $\mathcal{D}$ are totally geodesic hypersurfaces in $\bar{M}$. Let $M$ be a fixed integral leaf. The flow $\Phi: M \times \mathbb{R} \rightarrow \bar{M}$ generated by $Y$ takes isometrically $M=M_{0}$ to the leaf $M_{s}=\Phi_{s}(M)$ for any $s \in \mathbb{R}$, where $\Phi_{s}=\Phi(\cdot, s)$. The Riemannian connection in $M$ with the metric induced by its inclusion in $\bar{M}$ is denoted by $\nabla$.

Next, we consider an open, relatively compact domain $\Omega$ in $M$ with smooth boundary and we suppose that the immersion $\psi: \Omega \rightarrow \bar{M}$ is of the form

$$
\psi(x)=\psi_{u}(x)=\Phi(x, u(x)), \quad x \in \Omega,
$$

for some $\mathcal{C}^{\infty}$ function $u: \Omega \rightarrow \mathbb{R}$. In this case, the hypersurface

$$
\Sigma(u)=\psi(\Omega)
$$

is the Killing graph of the function $u$. 
Our first result gives a characterization of critical Killing graphs for the functional $\mathcal{F}_{f}$ in (2.3). Towards this end we assign local coordinates $y^{0}=s, y^{1}=$ $x^{1}, \ldots, y^{n}=x^{n}$ to a point $y=\Phi(x, s)$, if $x^{1}, \ldots, x^{n}$ are coordinates of the point $x \in \Omega$. We associate local coordinates $y^{0}, \ldots, y^{n}, \eta^{0}, \ldots, \eta^{n}$ to points $(y, \eta) \in T \bar{M}$ by setting

$$
\eta=\left.\eta^{\alpha} \frac{\partial}{\partial y^{\alpha}}\right|_{y} .
$$

In terms of these coordinates, the metric in $\bar{M}$ is written as

$$
\bar{g}_{\alpha \beta} d y^{\alpha} d y^{\beta}, \quad \text { for } \quad 0 \leq \alpha, \beta \leq n,
$$

where

$$
\bar{g}_{i j}=\sigma_{i j}, \quad \text { for } \quad 1 \leq i, j \leq n,
$$

are the components of the metric $\sigma$ induced on $\Omega$ by the inclusion $\Omega \subset \bar{M}$. Furthermore, we have

$$
\bar{g}_{00}=|Y|^{2}, \quad \bar{g}_{0 i}=0, \quad \text { for } \quad 1 \leq i \leq n .
$$

The components of the contravariant version of the metrics in $\bar{M}$ and $\Omega$ are respectively denoted by $\bar{g}^{\alpha \beta}$ and $\sigma^{i j}$.

Finally with $£_{Y}$ we will indicate the Lie derivative in the direction of $Y$. Recall that

$$
\operatorname{div}_{f} X=e^{f} \operatorname{div}\left(e^{-f} X\right),
$$

for $X \in \Gamma(T M)$ and $f \in \mathcal{C}^{\infty}(M)$. We are now ready to state:

Theorem 2.1. Suppose that $\left.£_{Y} F\right|_{\Sigma}=0$ for any Killing graph $\Sigma$ in $\bar{M}$, with $F$ as in (2.1). Then the Euler-Lagrange equation of the functional (2.3) is

$$
\operatorname{div}_{f} X-\left\langle X, \frac{\nabla \gamma}{2 \gamma}\right\rangle=0
$$

with $\gamma=|Y|^{-2}$ and

$$
X=\sigma^{i j} \frac{\partial F}{\partial \eta^{i}} \frac{\partial}{\partial x^{j}} .
$$

Moreover, a critical Killing graph $\psi$, for (2.3) constrained by some Lagrange multiplier $n \mathcal{H}$, satisfies the equation

$$
\operatorname{div}_{f} X-\left\langle X, \frac{\nabla \gamma}{2 \gamma}\right\rangle=-n \mathcal{H}
$$

In this case, we say that the Killing graph has prescribed generalized mean curvature $\mathcal{H}$. 
The proof of Theorem 2.1 is rather technical and, for the reader's convenience, is postponed to the Appendix. Now we discuss some important examples of this general formulation which we are going to consider in the sequel.

Example 2.2 (Killing graphs with prescribed mean curvature). We fix $F(y, \eta)=$ $|\eta|$ and we choose $f=0$ obtaining

$$
\mathcal{F}\left[\psi_{u}\right]=\int_{\Omega} \psi_{u}^{*} d \bar{M},
$$

that is, the induced Riemannian volume of the graph $\Sigma(u)$. With these choices we recover the classical setting of Killing graphs with prescribed mean curvature, [11]. As we will see next, the Euler-Lagrange equation (2.10) becomes

$$
\operatorname{div}\left(\frac{\nabla u}{W}\right)-\left\langle\frac{\nabla \gamma}{2 \gamma}, \frac{\nabla u}{W}\right\rangle=n H
$$

Indeed, since the flow of $Y$ preserves the length of vectors in $\bar{M}$, the Lagrangian $F$ is preserved along the flow lines of $Y$. In particular, $£_{Y} F=0$. Moreover we have

$$
\frac{\partial}{\partial \eta^{\alpha}}|\eta|=\frac{1}{|\eta|} \bar{g}_{\alpha \beta} \eta^{\beta} \text {. }
$$

Recall that the components of $\eta(y)=N$ along the graph $\Sigma(u)$ are given by

$$
\eta^{i}=-\frac{u^{i}}{W}, \quad \eta^{0}=\frac{\gamma}{W}
$$

where

$$
W=\sqrt{\gamma+|\nabla u|^{2}} .
$$

Hence we conclude that the components of $X$ are, in this case, given by $X^{i}=-\frac{u^{i}}{W}$, that is, $X=-\frac{\nabla u}{W}$. Therefore, (2.10) becomes (2.11).

Example 2.3 (Killing graphs with prescribed weighted mean curvature). Now we fix $F(y, \eta)=|\eta|$ and choose a general weight $e^{-f}$. This choice yields the weighted volume of Killing graphs defined by

$$
\mathcal{F}\left[\psi_{u}\right]=\int_{\Omega} e^{-f} \psi_{u}^{*} d \bar{M}
$$

whose critical points are $f$-minimal graphs in the sense that the weighted mean curvature

$$
H_{f}=H+\frac{1}{n}\langle\bar{\nabla} f, N\rangle
$$

vanishes on $\Sigma(u)$, where $H$ is the classical mean curvature of $\Sigma(u)$. In this case, the prescribed generalized mean curvature equation (2.10) becomes

$$
\operatorname{div}_{f}\left(\frac{\nabla u}{W}\right)-\left\langle\frac{\nabla \gamma}{2 \gamma}, \frac{\nabla u}{W}\right\rangle=n H_{f}
$$


Example 2.4 (Regularization of the 1-Laplacian). Given a constant $\lambda>0$, we define a positive definite symmetric tensor $g \in \Gamma\left(T^{*} \bar{M} \otimes T^{*} \bar{M}\right)$ by

$$
g(v, v)=\sigma\left(\pi_{*} v, \pi_{*} v\right)+\lambda\left\langle v, \frac{Y}{|Y|}\right\rangle^{2} .
$$

Here $\pi: \bar{M} \rightarrow M$ stands for the projection $\pi(y)=x$ if $y=\Phi(x, s)$. We then consider the perturbation of the classical volume Lagrangian $F(y, \eta)=|\eta|$ given by

$$
F_{\lambda}(y, \eta)=\sqrt{g(\eta, \eta)}
$$

Evaluating the corresponding functional at a Killing graph $\psi_{u}$ we have

$$
\mathcal{F}_{\lambda}\left[\psi_{u}\right]=\int_{\Omega} \sqrt{|\nabla u|^{2}+\lambda \gamma} d M
$$

where $\gamma=|Y|^{-2}$. The Euler-Lagrange equation (2.8) for this functional is

$$
\operatorname{div}\left(\frac{\nabla u}{\sqrt{|\nabla u|^{2}+\lambda \gamma}}\right)-\left\langle\frac{\nabla \gamma}{2 \gamma}, \frac{\nabla u}{\sqrt{|\nabla u|^{2}+\lambda \gamma}}\right\rangle=0
$$

Note that as $\lambda \rightarrow 0^{+}$this operator becomes the 1-Laplace operator that describes the level set formulation of the mean curvature flow in $M$ [15].

As pointed out by U. Clarenz and H. von der Mosel in [8], this kind of functional is used for numerical computations and surface processing involving the anisotropic mean curvature [7]. For more examples of integrands and applications in numerical analysis we refer to [13] and [14].

Example 2.5 (Killing graphs with constant anisotropic mean curvature). Consider the 1 -form in $\vec{M}$ defined by

$$
\Theta(\eta)=\langle Y(y), \eta\rangle, \quad \text { for } \quad \eta \in T_{y} \bar{M} \text {. }
$$

Note that $£_{Y} \Theta=0$. Given a differentiable function $a$, we consider the Lagrangian $F$ defined by

$$
F(y, \eta)=a\left(\Theta\left(\frac{\eta}{|\eta|}\right)\right) \sqrt{g(\eta, \eta)}
$$

where $g$ is the tensor defined in Example 2.4 above. In this case, and following the notation of Theorem 2.1, we obtain (see Section 5 for details)

$$
X=\left(a^{\prime}(\Theta) \Theta \sqrt{g(N, N)}-a(\Theta) \frac{1}{\sqrt{g(N, N)}}\right) \frac{\nabla u}{W},
$$


and therefore from (2.10) we have

$$
\begin{aligned}
& \operatorname{div}\left(\left(a(\Theta) \frac{1}{\sqrt{g(N, N)}}-a^{\prime}(\Theta) \Theta \sqrt{g(N, N)}\right) \frac{\nabla u}{W}\right) \\
& \quad-\left\langle\left(a(\Theta) \frac{1}{\sqrt{g(N, N)}}-a^{\prime}(\Theta) \Theta \sqrt{g(N, N)}\right) \frac{\nabla u}{W}, \frac{\nabla \gamma}{2 \gamma}\right\rangle=n H_{F},
\end{aligned}
$$

where $H_{F}$ is the anisotropic mean curvature of $\Sigma(u)$. This example generalizes to $\bar{M}$ the Lagrangians in $\mathbb{R}^{3}$ studied by $\mathrm{M}$. Koiso and B. Palmer in $[19,20]$.

It is worth pointing out that the different versions of the prescribed generalized mean curvature equation (2.10) that we have obtained in the examples above share some structural properties. In the Section 3 we will develop the analytical tools to deal with these equations with a unified approach. In Sections 4 and 5 we will apply these analytical results to draw geometric conclusions about the uniqueness and non-existence of solutions to these equations.

\section{A form of the weak maximum principle}

In this section, we prove a generalized form of the weak maximum principle for a class of divergence-form elliptic operators in a Riemannian manifold including the quasilinear geometric operators presented in Examples 2.2 to 2.5 in Section 2. The main novelty here is that the principal part of these operators might also depend on the point $x \in M$.

Throughout this section $(M,\langle\cdot, \cdot\rangle)$ denotes a complete Riemannian manifold. We let $r=r(x)$ be the geodesic distance in $M$ from some fixed origin $o \in M$ and we denote with $B_{R}=B_{R}(o)$ the geodesic ball centered at $o$ with radius $R$. We consider on $M$ the following operator

$$
L u=\operatorname{div}_{\phi}\left(|\nabla u|^{-1} \varphi(x,|\nabla u|) h(\nabla u, \cdot)^{\sharp}\right),
$$

where $\sharp$ denotes the musical isomorphism, $\phi \in \mathcal{C}^{\infty}(M), h$ is a positive definite symmetric 2-covariant tensor field on $M$ and $\varphi: M \times \mathbb{R}_{0}^{+} \rightarrow \mathbb{R}_{0}^{+}$satisfies $\varphi(\cdot, t) \in$ $\mathcal{C}^{0}(M)$ for every $t \in \mathbb{R}_{0}^{+}=[0,+\infty)$ and $\varphi(x, \cdot) \in \mathcal{C}^{0}\left(\mathbb{R}_{0}^{+}\right) \cap \mathcal{C}^{1}\left(\mathbb{R}^{+}\right)$for every $x \in M$, where $\mathbb{R}^{+}=(0,+\infty)$. Note that $L$ can be alternatively written as

$$
\begin{aligned}
L u & =e^{\phi} \operatorname{div}\left(e^{-\phi}|\nabla u|^{-1} \varphi(x,|\nabla u|) h(\nabla u, \cdot)^{\sharp}\right) \\
& =\operatorname{div}\left(|\nabla u|^{-1} \varphi(x,|\nabla u|) h(\nabla u, \cdot)^{\sharp}\right)-|\nabla u|^{-1} \varphi(x,|\nabla u|) h(\nabla u, \nabla \phi) .
\end{aligned}
$$

We assume that, for some continuous functions $h_{-}$and $h_{+}$defined on $\mathbb{R}_{0}^{+}$, the tensor $h$ satisfies the following bounds

$$
0<h_{-}(r) \leq h(X, X) \leq h_{+}(r)
$$


for every $X \in T_{x} M$, with $|X|=1$, and every $x \in \partial B_{r}$. Moreover, we also assume that $\varphi$ satisfies the following structure conditions:

i) $\varphi(x, 0)=0, \quad$ for every $x \in M$;

ii) $\varphi(x, t)>0, \quad$ on $M \times \mathbb{R}^{+}$;

iii) $\varphi(x, t) \leq A(x) t^{\delta}, \quad$ on $M \times \mathbb{R}^{+}$,

for some $\delta>0$ and $A(x) \in \mathcal{C}^{0}(M)$, with $A(x)>0$. Furthermore, assume that

$$
\inf _{M} \frac{h_{-}(r(x))}{h_{+}(r(x))} \frac{1}{A(x)^{1 / \delta}}=\frac{1}{C_{0}^{1 / \delta}}
$$

for some $C_{0}>0$.

Remark 3.1. Note that condition ii) in (3.3) is just an ellipticity condition for the operator $L$. In Sections 4 and 5 we verify that the operators given in the Examples 2.2 to 2.5 in Section 2 accomplish the requirements on $\varphi$ and $h$ for suitable choices of them.

Under these general assumptions on $L$, we give here a proof of a version of the weak maximum principle that we shall use in our geometric applications. Since the result is interesting in its own and could be used in different contexts, we present the proof in detail in order to make this section as self-contained as possible.

Theorem 3.2. Given $\varsigma, \mu \in \mathbb{R}$ we let

$$
\tau=\mu+(\varsigma-1)(1+\delta)
$$

and we assume that

$$
\varsigma \geq 0, \quad \varsigma-\tau>0 .
$$

Let $u \in \mathcal{C}^{2}(M)$ be a function such that

$$
\widehat{u}=\limsup _{r(x) \rightarrow+\infty} \frac{u(x)}{r(x)^{\varsigma}}<+\infty .
$$

Suppose that

$$
\liminf _{R \rightarrow+\infty} \frac{\log \int_{B_{R}} h_{+}(r) e^{-\phi}}{R^{5}-\tau}=d_{0}<+\infty .
$$

For $\xi \in \mathbb{R}$ suppose that the set

$$
\Omega_{\xi}=\{x \in M: u(x)>\xi\}
$$

is non-empty. Then

$$
\inf _{\Omega_{\xi}} \frac{(1+r(x))^{\mu}}{h_{+}(r(x))} L u(x) \leq \begin{cases}0 & \text { if } \varsigma=0 \\ C_{0} d_{0} \max \{\widehat{u}, 0\}^{\delta}(\varsigma-\tau)^{1+\delta} & \text { if } \varsigma>0 \text { and } \tau<0 \\ C_{0} d_{0} \max \{\widehat{u}, 0\}^{\delta} \varsigma^{\delta}(\varsigma-\tau) & \text { if } \varsigma>0 \text { and } \tau \geq 0 .\end{cases}
$$


Proof. We begin by observing that if $a$ is any constant and $u_{a}=u+a$, then

$$
L u_{a}=L u
$$

and

$$
\Omega_{\xi}=\left\{x \in M: u_{a}(x)>\xi+a\right\} .
$$

Furthermore, if $\varsigma>0$ then $\widehat{u}_{a}=\widehat{u}$, and if $\varsigma=0$ then $\widehat{u}_{a}=\widehat{u}+a$. So in order to estimate

$$
\inf _{\Omega_{\xi}} \frac{(1+r(x))^{\mu}}{h_{+}(r(x))} \operatorname{Lu}(x)
$$

we may replace $u$ with a suitable translate $u_{a}$. Next, fix $b>\max \{\widehat{u}, 0\}$. It is easy to see that there exists a constant $a$ such that

$$
\frac{u_{a}(x)}{(1+r(x))^{\varsigma}}<b \text { on } M
$$

and $u_{a}\left(x_{0}\right)>0$ for some $x_{0} \in M$. This is obvious if $u$ is bounded above and in particular, due to (3.7) if $\varsigma=0$. On the other hand, if $u$ is not bounded above, and therefore $\varsigma>0$, then by (3.7) there exists $\bar{R}>0$ such that

$$
\frac{u(x)}{(1+r(x))^{\varsigma}}<b \text { on } M \backslash B_{\bar{R}},
$$

and it is clear that there exists $a \in \mathbb{R}$ such that $u_{a}\left(x_{0}\right)>0$ for some $x_{0} \in \bar{B}_{\bar{R}}$ and $\frac{u_{a}(x)}{(1+r(x))^{\zeta}}<b$ on $\bar{B}_{\bar{R}}$ and on all of $M$. We will assume that a constant $a$ has been selected in such a way that (3.11) holds. In accordance to the observation made above, we are going to replace $u$ with $u_{a}$ and, for the ease of notation, we suppress the subscript $a$. Furthermore, if $\xi_{1} \leq \xi$ and

$$
\inf _{\Omega_{\xi}} \frac{(1+r(x))^{\mu}}{h_{+}(r(x))} L u(x) \leq D
$$

then

$$
\inf _{\Omega_{\xi_{1}}} \frac{(1+r(x))^{\mu}}{h_{+}(r(x))} L u(x) \leq D
$$

so that, without loss of generality, we may suppose $\xi \geq 0$. Next, let

$$
K=\inf _{\Omega_{\xi}} \frac{(1+r(x))^{\mu}}{h_{+}(r(x))} L u(x)
$$

and suppose $K>0$, otherwise there is nothing to prove. In this case $u$ is nonconstant on any component of $\Omega_{\xi}$ and

$$
\frac{(1+r(x))^{\mu}}{h_{+}(r(x))} L u(x) \geq K>0 \text { on } \Omega_{\xi} .
$$


We fix $\theta \in(1 / 2,1)$ and we choose $R_{0}>0$ large enough so that $B_{R_{0}} \cap \Omega_{\xi} \neq \emptyset$ and $|\nabla u| \not \equiv 0$ on it. Given $R>R_{0}$ we let $\psi \in \mathcal{C}^{\infty}(M)$ be a cut off function such that $0 \leq \psi \leq 1$ and

$$
\begin{cases}\psi \equiv 1 & \text { on } B_{\theta R} \\ \psi \equiv 0 & \text { on } M \backslash B_{R} \\ |\nabla \psi| \leq \frac{C}{R(1-\theta)} & \end{cases}
$$

for some constant $C>0$. Let also $\lambda \in \mathcal{C}^{1}(\mathbb{R})$ and $F(v, r) \in \mathcal{C}^{1}\left(\mathbb{R}^{2}\right)$ be such that $0 \leq \lambda \leq 1$ and

$$
\begin{cases}\lambda=0 & \text { on }(-\infty, \xi] \\ \lambda>0, \lambda^{\prime} \geq 0 & \text { on }(\xi,+\infty)\end{cases}
$$

and

$$
F(v, r)>0, \quad \frac{\partial F}{\partial v}(v, r)<0 \text { on } \mathbb{R}_{0}^{+} \times \mathbb{R}_{0}^{+} .
$$

Finally, we let $Z$ be the vector field defined on $\Omega_{\xi}$ by

$$
Z=\psi^{1+\delta} \lambda(u) F(v, r) e^{-\phi}|\nabla u|^{-1} \varphi(x,|\nabla u|) h(\nabla u, \cdot)^{\sharp}
$$

where $v$ is given by

$$
v=\alpha(1+r)^{\varsigma}-u
$$

and $\alpha>b$ is a constant so that $v>0$ on $\Omega_{\xi}$. Indeed, according to (3.11) and the assumption $\xi \geq 0$, so that $u>0$ on $\Omega \xi$, we have

$$
(\alpha-b)(1+r)^{\varsigma} \leq v \leq \alpha(1+r)^{\varsigma} \quad \text { on } \Omega_{\xi} .
$$

Note that $Z$ vanishes on $\partial\left(\Omega_{\xi} \cap B_{R}\right)$ and it extends to a continuous vector field on the whole of $M$ by defining it to be zero in the complement of $\Omega_{\xi} \cap B_{R}$. In what follows we proceed as if $Z$ were of class $\mathcal{C}^{1}$. This will allow us to better explain the underlying reasons of the argument of the proof. Having done this, it is an easy matter to provide a proof for the general continuous case using the weak formulation of the divergence theorem (see for instance [24] for a similar case).

We now compute the divergence of $Z$. Note that, from iii) in (3.3), we have

$$
t \varphi(x, t) \geq A(x)^{-1 / \delta} \varphi(x, t)^{1+1 / \delta} \quad \text { on } M \times \mathbb{R}_{0}^{+} .
$$

Furthermore, from the properties (3.2) of $h$

$$
|h(\nabla u, \nabla v)| \leq \sqrt{h(\nabla u, \nabla u)} \sqrt{h(\nabla v, \nabla v)} \leq h_{+}(r(x))|\nabla u||\nabla v| .
$$


We compute

$$
\begin{aligned}
e^{\phi} \operatorname{div} Z= & \psi^{1+\delta} \lambda(u) F(v, r) L u \\
& +(1+\delta) \psi^{\delta} \lambda(u) F(v, r)|\nabla u|^{-1} \varphi(x,|\nabla u|) h(\nabla u, \nabla \psi) \\
& +\psi^{1+\delta} \lambda^{\prime}(u) F(v, r)|\nabla u|^{-1} \varphi(x,|\nabla u|) h(\nabla u, \nabla u) \\
& +\psi^{1+\delta} \lambda(u) \frac{\partial F}{\partial v}(v, r)|\nabla u|^{-1} \varphi(x,|\nabla u|) h(\nabla u, \nabla v) \\
& +\psi^{1+\delta} \lambda(u) \frac{\partial F}{\partial r}(v, r)|\nabla u|^{-1} \varphi(x,|\nabla u|) h(\nabla u, \nabla r),
\end{aligned}
$$

that yields

$$
\begin{aligned}
e^{\phi} \operatorname{div} Z \geq & \psi^{1+\delta} \lambda(u) F(v, r) K(1+r)^{-\mu} h_{+}(r) \\
& -(1+\delta) \psi^{\delta} \lambda(u) F(v, r) \varphi(x,|\nabla u|) h_{+}(r)|\nabla \psi| \\
& +\psi^{1+\delta} \lambda(u) \frac{\partial F}{\partial v}(v, r)|\nabla u|^{-1} \varphi(x,|\nabla u|) h\left(\nabla u, \alpha \varsigma(1+r)^{\varsigma-1} \nabla r-\nabla u\right) \\
& +\psi^{1+\delta} \lambda(u) \frac{\partial F}{\partial r}(v, r)|\nabla u|^{-1} \varphi(x,|\nabla u|) h(\nabla u, \nabla r),
\end{aligned}
$$

where to obtain the last inequality we have used (3.2), (3.12), (3.13), (3.16) and (3.19). Using now (3.2), (3.14) and (3.18) we obtain

$$
\begin{aligned}
e^{\phi} \operatorname{div} Z \geq & -(1+\delta) \psi^{\delta} \lambda(u) F(v, r) \varphi(x,|\nabla u|) h_{+}(r)|\nabla \psi| \\
& +\psi^{1+\delta} \lambda(u) F(v, r) K(1+r)^{-\mu} h_{+}(r) \\
& -\psi^{1+\delta} \lambda(u) \frac{\partial F}{\partial v}(v, r) \varphi(x,|\nabla u|)|\nabla u| h_{-}(r) \\
& +\alpha \varsigma(1+r)^{\varsigma-1} \psi^{1+\delta} \lambda(u) \frac{\partial F}{\partial v}(v, r)|\nabla u|^{-1} \varphi(x,|\nabla u|) h(\nabla u, \nabla r) \\
& +\psi^{1+\delta} \lambda(u) \frac{\partial F}{\partial r}(v, r)|\nabla u|^{-1} \varphi(x,|\nabla u|) h(\nabla u, \nabla r)
\end{aligned}
$$

and therefore

$$
\begin{aligned}
e^{\phi} \operatorname{div} Z \geq & -(1+\delta) \psi^{\delta} \lambda(u) F(v, r) \varphi(x,|\nabla u|) h_{+}(r)|\nabla \psi| \\
& +\psi^{1+\delta} \lambda(u) F(v, r) K(1+r)^{-\mu} h_{+}(r) \\
& +\psi^{1+\delta} \lambda(u)\left|\frac{\partial F}{\partial v}(v, r)\right| \frac{h_{-}(r)}{A(x)^{1 / \delta}} \varphi(x,|\nabla u|)^{1+1 / \delta} \\
& -\psi^{1+\delta} \lambda(u)\left|\frac{\partial F}{\partial v}(v, r)\right| \alpha \varsigma(1+r)^{\varsigma^{-1}}|\nabla u|^{-1} \varphi(x,|\nabla u|) h(\nabla u, \nabla r) \\
& +\psi^{1+\delta} \lambda(u)\left|\frac{\partial F}{\partial v}(v, r)\right| \frac{\frac{\partial F}{\partial r}(v, r)}{\left|\frac{\partial F}{\partial v}(v, r)\right|}|\nabla u|^{-1} \varphi(x,|\nabla u|) h(\nabla u, \nabla r) .
\end{aligned}
$$


Hence, we have

$$
\begin{aligned}
e^{\phi} \operatorname{div} Z \geq & -(1+\delta) \psi^{\delta} \lambda(u) F(v, r) \varphi(x,|\nabla u|) h_{+}(r)|\nabla \psi| \\
& +\psi^{1+\delta} \lambda(u)\left|\frac{\partial F}{\partial v}(v, r)\right| B(x, \nabla u, r),
\end{aligned}
$$

where

$$
\begin{aligned}
B(x, \nabla u, r)= & \frac{h_{-}(r)}{A(x)^{1 / \delta}} \varphi(x,|\nabla u|)^{1+1 / \delta}+\frac{F(v, r)}{\left|\frac{\partial F}{\partial v}(v, r)\right|} K(1+r)^{-\mu} h_{+}(r) \\
& +\left(x \frac{\frac{\partial F}{\partial r}(v, r)}{\left|\frac{\partial F}{\partial v}(v, r)\right|}-\alpha \varsigma(1+r)^{\varsigma^{-1}}\right)|\nabla u|^{-1} \varphi(x,|\nabla u|) h(\nabla u, \nabla r) .
\end{aligned}
$$

Using (3.4) we obtain

$$
\begin{aligned}
& B(x, \nabla u, r) \geq\left(\frac{1}{C_{0}^{1 / \delta}} \varphi(x,|\nabla u|)^{1+1 / \delta}+\frac{F(v, r)}{\left|\frac{\partial F}{\partial v}(v, r)\right|} K(1+r)^{-\mu}\right) h_{+}(r) \\
& +\left(\frac{\frac{\partial F}{\partial r}(v, r)}{\left|\frac{\partial F}{\partial v}(v, r)\right|}-\alpha \varsigma(1+r)^{\varsigma-1}\right)|\nabla u|^{-1} \varphi(x,|\nabla u|) h(\nabla u, \nabla r) .
\end{aligned}
$$

Next, we consider different cases.

Case 1: $\tau<0$. We choose

$$
F(v, r)=e^{-q v(1+r)^{-\tau}}
$$

where $q>0$ is a constant that will be specified later. We use $(3.16),(3.17),(3.6)$ and $\alpha>0$ to obtain

$$
0 \geq \frac{\frac{\partial F}{\partial r}(v, r)}{\left|\frac{\partial F}{\partial v}(v, r)\right|}-\alpha \varsigma(1+r)^{\varsigma-1} \geq-\alpha(\varsigma-\tau)(1+r)^{\varsigma^{-1}}
$$

on $\Omega_{\xi}$ and

$$
\frac{F(v, r)}{\left|\frac{\partial F}{\partial v}(v, r)\right|}=\frac{1}{q}(1+r)^{\tau}
$$

Also note that

$$
|\nabla u|^{-1} \varphi(x,|\nabla u|) h(\nabla u, \nabla r) \leq \varphi(x,|\nabla u|) h_{+}(r) .
$$


Inserting (3.23) and (3.24) into (3.22) and using (3.5) we deduce

$$
\begin{aligned}
B(x, \nabla u, r) \geq & {\left[\frac{1}{C_{0}^{1 / \delta}} \varphi(x,|\nabla u|)^{1+1 / \delta}+\frac{K}{q}(1+r)^{(\varsigma-1)(1+\delta)}\right.} \\
& \left.-\alpha(\varsigma-\tau)(1+r)^{\varsigma-1} \varphi(x,|\nabla u|)\right] h_{+}(r) .
\end{aligned}
$$

At this point we need to estimate the right-hand side of (3.25) so as to have

$$
B(x, \nabla u, r) \geq \Lambda \varphi(x,|\nabla u|)^{1+1 / \delta} h_{+}(r)
$$

where $\Lambda$ is a positive constant independent of $\nabla u, r$ and $x$. For this purpose we use the next lemma whose proof is a calculus exercise.

Lemma 3.3. Let $\delta, \varrho, \beta, \omega$ be positive constants and let $f$ be the function defined on $\mathbb{R}_{0}^{+}$by $f(s)=\omega s^{1+1 / \delta}-\beta s+\varrho$. Then the inequality $f(s) \geq \Lambda s^{1+1 / \delta}$ holds on $\mathbb{R}_{0}^{+}$provided

$$
\Lambda \leq \omega-\frac{\delta \beta^{1+1 / \delta}}{(1+\delta)^{1+1 / \delta} \varrho^{1 / \delta}} .
$$

Applying Lemma 3.3 with $s=\varphi(x,|\nabla u|)$ and $x$ fixed it is easy to verify that (3.25) holds independently of $x$ if we can choose a positive $\Lambda$ such that

$$
\Lambda \leq \frac{1}{C_{0}^{1 / \delta}}-\frac{q^{1 / \delta} \delta(\alpha(\varsigma-\tau))^{1+1 / \delta}}{(1+\delta)^{1+1 / \delta} K^{1 / \delta}},
$$

where $r=r(x)$. Thus, if $\vartheta \in(0,1)$ and we choose

$$
q=\frac{\vartheta^{\delta} K(1+\delta)^{1+\delta}}{C_{0} \delta^{\delta}(\alpha(\varsigma-\tau))^{1+\delta}} \quad \text { and } \quad \Lambda=\frac{1-\vartheta}{C_{0}^{1 / \delta}}
$$

then $\Lambda>0$ and it satisfies (3.27).

We insert (3.26) and the expression for $\partial F / \partial v$ into (3.20) to obtain

$$
\begin{aligned}
\operatorname{div} Z \geq e^{-\phi} & \left(-(1+\delta) \psi^{\delta} \lambda(u) F(v, r) \varphi(x,|\nabla u|) h_{+}(r)|\nabla \psi|\right. \\
& \left.+q \Lambda \psi^{1+\delta} \lambda(u)(1+r)^{-\tau} F(v, r) \varphi(x,|\nabla u|)^{1+1 / \delta} h_{+}(r)\right) .
\end{aligned}
$$

We integrate this inequality on $\Omega_{\xi} \cap B_{R}$, apply the divergence theorem and recall that $Z$ vanishes on $\partial\left(\Omega_{\xi} \cap B_{R}\right)$ to obtain

$$
\begin{aligned}
& \frac{q \Lambda}{1+\delta} \int_{\Omega_{\xi} \cap B_{R}} \psi^{1+\delta} \lambda(u)(1+r)^{-\tau} F(v, r) \varphi(x,|\nabla u|)^{1+1 / \delta} h_{+}(r) e^{-\phi} \\
\leq & \int_{\Omega_{\xi} \cap B_{R}} \psi^{\delta} \lambda(u) F(v, r) \varphi(x,|\nabla u|) h_{+}(r)|\nabla \psi| e^{-\phi} .
\end{aligned}
$$


We write

$$
\psi^{\delta} \lambda(u) F(v, r) \varphi(x,|\nabla u|) h_{+}(r)|\nabla \psi| e^{-\phi}=g_{1} g_{2}
$$

with

$$
g_{1}=\left(\lambda(u) F(v, r) h_{+}(r)\right)^{\frac{1}{1+\delta}}|\nabla \psi|(1+r)^{\frac{\tau \delta}{1+\delta}} e^{-\frac{1}{1+\delta} \phi}
$$

and

$$
\left.g_{2}=\left(\lambda(u) F(v, r) h_{+}(r)\right)^{\frac{\delta}{1+\delta}} \psi^{\delta} \varphi(x,|\nabla u|)\right)(1+r)^{-\frac{\tau \delta}{1+\delta}} e^{-\frac{\delta}{1+\delta} \phi} .
$$

Applying Hölder's inequality with conjugate exponents $1+\delta$ and $1+1 / \delta$ to the integral on the right-hand side we obtain

$$
\begin{aligned}
& \int_{\Omega_{\xi} \cap B_{R}} \psi^{\delta} \lambda(u) F(v, r) \varphi(x,|\nabla u|) h_{+}(r)|\nabla \psi| e^{-\phi} \\
& \leq\left(\int_{\Omega_{\xi} \cap B_{R}} \lambda(u) F(v, r) h_{+}(r)|\nabla \psi|^{1+\delta}(1+r)^{\tau \delta} e^{-\phi}\right)^{\frac{1}{1+\delta}} \\
& \left.\quad \times\left(\int_{\Omega_{\xi} \cap B_{R}} \lambda(u) F(v, r) h_{+}(r) \psi^{1+\delta} \varphi(x,|\nabla u|)\right)^{\frac{1+\delta}{\delta}}(1+r)^{-\tau} e^{-\phi}\right)^{\frac{\delta}{1+\delta}},
\end{aligned}
$$

and after some simplification from the above we get

$$
\begin{aligned}
& \left(\frac{q \Lambda}{1+\delta}\right)^{1+\delta} \int_{\Omega_{\xi} \cap B_{R}} \psi^{1+\delta} \lambda(u)(1+r)^{-\tau} F(v, r) \varphi(x,|\nabla u|)^{1+1 / \delta} h_{+}(r) e^{-\phi} \\
\leq & \int_{\Omega_{\xi} \cap B_{R}} \lambda(u) F(v, r)(1+r)^{\tau \delta}|\nabla \psi|^{1+\delta} h_{+}(r) e^{-\phi} .
\end{aligned}
$$

Let $R>2 R_{0}$; then $\theta R>R / 2>R_{0}$ and using the properties of $\lambda$ and $\psi$ we deduce

$$
\begin{aligned}
E & =\left(\frac{q \Lambda}{1+\delta}\right)^{1+\delta} \int_{\Omega_{\xi} \cap B_{R_{0}}} \lambda(u) F(v, r) \varphi(x,|\nabla u|)^{1+1 / \delta} h_{+}(r) e^{-\phi} \\
& \leq C^{1+\delta}(1+\theta R)^{\tau \delta}[(1-\theta) R]^{-(1+\delta)} \int_{\Omega_{\xi} \cap\left(B_{R} \backslash B_{\theta R}\right)} F(v, r) h_{+}(r) e^{-\phi} .
\end{aligned}
$$

Using (3.17) for $v$ and the expression of $F$ on $\Omega_{\xi} \cap\left(B_{R} \backslash B_{\theta R}\right)$

$$
F(v, r) \leq e^{-q(\alpha-b)(1+\theta R)^{\varsigma-\tau}},
$$

thus from (3.29)

$$
E \leq \widehat{C} R^{\delta \tau-1-\delta} e^{-q(\alpha-b)(1+\theta R)^{\varsigma-\tau}} \int_{B_{R}} h_{+}(r) e^{-\phi}
$$


for some constant $\widehat{C}>0$. Now observe that, since $|\nabla u| \not \equiv 0$ on $\Omega_{\xi} \cap B_{R_{0}}$, it follows that $E>0$. From assumption (3.8), for every fixed $d>d_{0}$ there exists a strictly increasing sequence $R_{k} \nearrow+\infty$ with $R_{1}>2 R_{0}$ and such that

$$
\log \int_{B_{R_{k}}} h_{+}(r) e^{-\phi} \leq d R_{k}^{\zeta-\tau}
$$

and from the above inequality with $R=R_{k}$ we obtain

$$
\begin{aligned}
0<E & \leq \widehat{C} R_{k}^{\delta \tau-1-\delta} e^{-q(\alpha-b)\left(1+\theta R_{k}\right)^{5-\tau}} \int_{B_{R_{k}}} h_{+}(r) e^{-\phi} \\
& \leq \widehat{C} R_{k}^{\delta \tau-1-\delta} e^{d R_{k}^{\zeta-\tau}-q(\alpha-b)\left(1+\theta R_{k}\right)^{\zeta-\tau}}
\end{aligned}
$$

where the constant $\widehat{C}>0$ is independent of $k$. In order for this inequality to hold for every $k$, we must have

$$
d \geq(\alpha-b) q \theta^{\varsigma-\tau}
$$

hence, letting $\theta \rightarrow 1$,

$$
d \geq(\alpha-b) q .
$$

We set $\alpha=t b, t>1$, and we insert the choice (3.28) of $q$ in the above inequality, solve with respect to $K$, and let $\vartheta \nearrow 1$ to obtain

$$
K \leq C_{0} d b^{\delta}(\varsigma-\tau)^{1+\delta} \frac{\delta^{\delta}}{(1+\delta)^{1+\delta}} \frac{t^{1+\delta}}{t-1} .
$$

Therefore, minimizing with respect to $t>1$ and letting $d \rightarrow d_{0}, b \rightarrow \max \{\widehat{u}, 0\}$, we obtain

$$
K \leq C_{0} d_{0} \max \{\widehat{u}, 0\}^{\delta}(\varsigma-\tau)^{1+\delta} .
$$

In other words,

$$
\inf _{\Omega_{\xi}} \frac{(1+r)^{\mu}}{h_{+}(r)} L u \leq C_{0} d_{0} \max \{\widehat{u}, 0\}^{\delta}(\varsigma-\tau)^{1+\delta} .
$$

This finishes the proof when $\varsigma>0$ and $\tau<0$.

For $\varsigma=0$ (and necessarily $\tau<0$ by (3.6)) we can improve the above estimate as follows. We apply (3.31) to the function $u-\widehat{u}$ on the set

$$
\{x \in M: u(x)-\widehat{u}>\xi-\widehat{u}\}=\Omega_{\xi},
$$

observing that $\widehat{u-\widehat{u}}=0$ and that $L u=L(u-\widehat{u})$, to obtain

$$
\inf _{\Omega_{\xi}} \frac{(1+r)^{\mu}}{h_{+}(r)} L u \leq 0 .
$$


Case 2: $\tau \geq 0$ (and necessarily $\varsigma>0$ by (3.6)). We choose

$$
F(v, r)=F(v)=e^{-q v^{(\varsigma-\tau) / \varsigma}}
$$

where $q>0$ is a constant to be specified later. Noting that the exponent of $v$ is positive by (3.6), a computation yields

$$
\frac{\partial F}{\partial v}(v, r)=-q \frac{\varsigma-\tau}{\varsigma} v^{-\tau / \varsigma} F(v)<0,
$$

while clearly, $\partial F / \partial r \equiv 0$.

Using estimate (3.22), recalling that $v \geq(\alpha-b)(1+r)^{\varsigma}$ (see (3.17)), and proceeding as in Case 1, we estimate

$$
\begin{aligned}
B(x, \nabla u, r) \geq & {\left[\frac{1}{C_{0}^{1 / \delta}} \varphi(x,|\nabla u|)^{1+1 / \delta}-\alpha \varsigma(1+r)^{\varsigma-1} \varphi(x,|\nabla u|)\right.} \\
& \left.+\frac{\varsigma}{q(\varsigma-\tau)}(\alpha-b)^{\tau / \varsigma} K(1+r)^{(\varsigma-1)(1+\delta)}\right] h_{+}(r) .
\end{aligned}
$$

According to Lemma 3.3, for every $r \geq 0$ fixed, the right-hand side of the above inequality is bounded from below by $\Lambda \varphi(x,|\nabla u|)^{1+1 / \delta} h_{+}(r)$ provided

$$
\Lambda \leq \frac{1}{C_{0}^{1 / \delta}}-\frac{q^{1 / \delta} \delta(\alpha \varsigma)^{1+1 / \delta}(\varsigma-\tau)^{1 / \delta}}{(1+\delta)^{1+1 / \delta}(K \varsigma)^{1 / \delta}(\alpha-b)^{\tau /(\delta \varsigma)}} .
$$

Since the right-hand side of the above inequality is independent of $r$, for every such $\Lambda$ we have $B(x, \nabla u, r) \geq \Lambda \varphi(x,|\nabla u|)^{1+1 / \delta} h_{+}(r)$. In particular, if $\vartheta \in(0,1)$ and we choose

$$
q=\frac{\vartheta^{\delta} K \varsigma(1+\delta)^{1+\delta}(\alpha-b)^{\tau / \varsigma}}{C_{0} \delta^{\delta}(\alpha \varsigma)^{1+\delta}(\varsigma-\tau)} \quad \text { and } \quad \Lambda=\frac{1-\vartheta}{C_{0}^{1 / \delta}}
$$

then $\Lambda>0$ and it satisfies (3.33). Substituting into (3.20), and using the expression for $\partial F / \partial v$, we deduce that

$$
\begin{aligned}
\operatorname{div} Z \geq e^{-\phi}( & -(1+\delta) \psi^{\delta} \lambda(u) F(v) \varphi(x,|\nabla u|) h_{+}(r)|\nabla \psi| \\
& \left.+q \frac{\varsigma-\tau}{\varsigma} \Lambda \psi^{1+\delta} \lambda(u) v^{\tau / \varsigma} F(v) \varphi(x,|\nabla u|)^{1+1 / \delta} h_{+}(r)\right) .
\end{aligned}
$$

We now proceed as in Case 1, repeating, with minor adaptations, the arguments that lead to (3.29), to conclude instead that

$$
\begin{aligned}
0<E & =\int_{\Omega_{\xi} \cap B_{R_{0}}} \lambda(u) F(v) \varphi(x,|\nabla u|)^{1+1 / \delta} h_{+}(r) e^{-\phi} \\
& \leq \widehat{C}\left(1+\theta R_{k}\right)^{\tau \delta}\left[(1-\theta) R_{k}\right]^{-(1+\delta)} \int_{\Omega_{\xi} \cap\left(B_{R_{k}} \backslash B_{\theta R_{k}}\right)} F(v) h_{+}(r) e^{-\phi},
\end{aligned}
$$


where $\widehat{C}$ is a constant independent of $k$ and $\theta$. Using the inequality

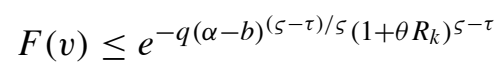

valid on $\Omega_{\xi} \cap\left(B_{R_{k}} \backslash B_{\theta R_{k}}\right)$, and (3.30), we conclude that for every $k$ we have

$$
0<E \leq \widehat{C} R_{k}^{\delta \tau-1-\delta} e^{d R_{k}^{\varsigma-\tau}-q(\alpha-b)^{(\varsigma-\tau) / \varsigma}\left(1+\theta R_{k}\right)^{\varsigma-\tau}} .
$$

Again, this forces

$$
d \geq q(\alpha-b)^{(\varsigma-\tau) / \varsigma} \theta^{\varsigma-\tau} .
$$

Therefore, setting $\alpha=t b, t>1$, letting $\theta \nearrow 1$, inserting the value of $q$ given by (3.34), solving with respect to $K$ and letting $\vartheta \nearrow 1, d \searrow d_{0}, b \searrow \max \{\widehat{u}, 0\}$, we obtain

$$
K \leq C_{0} d_{0} \max \{\widehat{u}, 0\}^{\delta} \frac{(\delta \varsigma)^{\delta}(\varsigma-\tau)}{(1+\delta)^{1+\delta}} \frac{t^{1+\delta}}{t-1}
$$

hence, again minimizing with respect to $t>1$, we conclude that

$$
K \leq C_{0} d_{0} \max \{\widehat{u}, 0\}^{\delta} \varsigma^{\delta}(\varsigma-\tau) .
$$

In other words,

$$
\inf _{\Omega_{\xi}} \frac{(1+r)^{\mu}}{h_{+}(r)} L u \leq C_{0} d_{0} \max \{\widehat{u}, 0\}^{\delta} \varsigma^{\delta}(\varsigma-\tau) .
$$

This finishes the proof when $\tau \geq 0$.

\subsection{A criterion for parabolicity}

In this subsection we derive a parabolicity criterion for the operator

$$
L u=e^{\phi} \operatorname{div}\left(e^{-\phi}|\nabla u|^{-1} \varphi(x,|\nabla u|) h(\nabla u, \cdot)^{\sharp}\right)
$$

defined in (3.1). Recall that a Riemannian manifold $(M,\langle\cdot, \cdot\rangle)$ is said to be parabolic if the only subharmonic functions on $M$ which are bounded from above are constant; that is, if the only solutions $u$ of the inequality $\Delta u \geq 0$ which are bounded from above are constant. Following this terminology, we say that the Riemannian manifold $(M,\langle\cdot, \cdot\rangle)$ is $L$-parabolic if the only solutions $u$ of the inequality $L u \geq 0$ which are bounded from above are constant.

The following result establishes a sufficient condition for $L$-parabolicity of a complete manifold $(M,\langle\cdot, \cdot\rangle)$

Theorem 3.4. Let $(M,\langle\cdot, \cdot\rangle)$ be a complete manifold, $o \in M$ a fixed origin and $r(x)=\operatorname{dist}_{M}(x, o)$. Let $L$ be the operator defined in (3.36) with $h$ and $\varphi$ satisfying the assumptions (3.2), (3.3) and (3.4) above. Let $h_{+}(r)$ be defined in (3.2). If

$$
\frac{1}{\left(\int_{\partial B_{t}} h_{+}(r) e^{-\phi}\right)^{\frac{1}{\delta}}} \notin L^{1}(+\infty)
$$

then $(M,\langle\cdot, \cdot\rangle)$ is L-parabolic. 
The proof of Theorem 3.4 requires the following technical lemma

Lemma 3.5. Let $L$ be the operator defined in (3.36) with $h$ and $\varphi$ satisfying the assumptions (3.2), (3.3) and (3.4). Let $\kappa \in \mathcal{C}^{0}(\mathbb{R})$ and let $u$ be a non-constant $\mathcal{C}^{1}$ solution on $M$ of the differential inequality

$$
L u \geq|\nabla u|^{-1} \varphi(x,|\nabla u|) h(\nabla u, \nabla u) \kappa(u) .
$$

Assume that there exist functions $\alpha \in \mathcal{C}^{1}(I)$ and $\beta \in \mathcal{C}^{0}(I)$ defined on an interval $I \supset u(M)$ such that

$$
\begin{aligned}
& \alpha(u) \geq 0, \\
& \alpha^{\prime}(u)+\kappa(u) \alpha(u) \geq \beta(u)>0
\end{aligned}
$$

on $M$. Then, there exist $R_{0}>0$ depending only on $u$ and a constant $C>0$ independent of $\alpha$ and $\beta$, such that, for any $r>R \geq R_{0}$

$$
\begin{aligned}
& \left(\int_{B_{r}} \beta(u) \varphi(x,|\nabla u|)|\nabla u| h_{-}(r) e^{-\phi}\right)^{-1} \\
\geq & C\left(\int_{R}^{r}\left(\int_{\partial B_{r}} e^{-\phi} h_{+}(r) \frac{\alpha(u)^{1+\delta}}{\beta(u)^{\delta}}\right)^{-\frac{1}{\delta}}\right)^{\delta} .
\end{aligned}
$$

Proof. We consider the vector field

$$
Z=\alpha(u) e^{-\phi}|\nabla u|^{-1} \varphi(x,|\nabla u|) h(\nabla u, \cdot)^{\sharp} .
$$

We compute the distributional divergence of $Z$ and we use our assumptions on $\alpha, \beta$ and (3.38) to obtain

$$
\begin{aligned}
\operatorname{div} Z & \geq\left(\alpha^{\prime}(u)+\kappa(u) \alpha(u)\right) e^{-\phi}|\nabla u|^{-1} \varphi(x,|\nabla u|) h(\nabla u, \nabla u) \\
& \geq \beta(u) e^{-\phi}|\nabla u|^{-1} \varphi(x,|\nabla u|) h(\nabla u, \nabla u) .
\end{aligned}
$$

Using (3.2) we immediately obtain

$$
\operatorname{div} Z \geq \beta(u) e^{-\phi}|\nabla u| \varphi(x,|\nabla u|) h_{-}(r) .
$$

Integrating over $B_{t}$ and applying the divergence theorem gives

$$
\int_{\partial B_{t}}\langle Z, \nabla r\rangle e^{-\phi} \geq \int_{B_{t}} \beta(u)|\nabla u| \varphi(x,|\nabla u|) h_{-}(r) e^{-\phi} .
$$

On the other hand, using Cauchy-Schwarz inequality and (3.2), we have

$$
\int_{\partial B_{t}}\langle Z, \nabla r\rangle e^{-\phi} \leq \int_{\partial B_{t}} \alpha(u) \varphi(x,|\nabla u|) h_{+}(r) e^{-\phi} .
$$


We observe that assumption iii) on $\varphi$ in (3.3) implies (see (3.18))

$$
t \varphi(x, t) \geq A(x)^{-\frac{1}{\delta}} \varphi(x, t)^{1+\frac{1}{\delta}} .
$$

Hence,

$$
\begin{aligned}
& \alpha(u) \varphi(x,|\nabla u|) h_{+}(r) e^{-\phi} \\
\leq & A(x)^{\frac{1}{1+\delta}} \frac{\alpha(u)}{\beta(u)^{\frac{\delta}{1+\delta}}} \frac{h_{+}(r)}{h_{-}(r)^{\frac{\delta}{1+\delta}}} e^{-\phi \frac{1}{1+\delta}}(|\nabla u| \varphi(x,|\nabla u|))^{\frac{\delta}{1+\delta}} e^{-\phi \frac{\delta}{1+\delta}} \beta(u)^{\frac{\delta}{1+\delta}} h_{-}(r)^{\frac{\delta}{1+\delta}} .
\end{aligned}
$$

Thus, applying Hölder's inequality with conjugate exponents $p=1+\delta$ and $q=$ $1+\frac{1}{\delta}$ we obtain

$$
\begin{aligned}
& \int_{\partial B_{t}}\langle Z, \nabla r\rangle e^{-\phi} \leq \int_{\partial B_{t}} \alpha(u) \varphi(x,|\nabla u|) h_{+}(r) e^{-\phi} \\
\leq & \left(\int_{\partial B_{t}} A(x) \frac{\alpha(u)^{1+\delta}}{\beta(u)^{\delta}} \frac{h_{+}(r)^{1+\delta}}{h_{-}(r)^{\delta}} e^{-\phi}\right)^{\frac{1}{1+\delta}}\left(\int_{\partial B_{t}}|\nabla u| \varphi(x,|\nabla u|) \beta(u) h_{-}(r) e^{-\phi}\right)^{\frac{\delta}{1+\delta}} .
\end{aligned}
$$

We set

$$
G(R)=\int_{B_{R}} \beta(u) h_{-}(r)|\nabla u| \varphi(x,|\nabla u|) e^{-\phi}
$$

and we observe that, since $u$ is non constant, there exists $R_{0}>0$ sufficiently large such that, for any $R \geq R_{0}$, it holds that $G(R)>0$. Using the coarea formula and putting together (3.44) and (3.47) we obtain

$$
G(R)^{\frac{1+\delta}{\delta}} \leq G^{\prime}(R)\left(\int_{\partial B_{R}} A(x) \frac{\alpha(u)^{1+\delta}}{\beta(u)^{\delta}} \frac{h_{+}(r)^{\delta}}{h_{-}(r)^{\delta}} h_{+}(r) e^{-\phi}\right)^{\frac{1}{\delta}}
$$

for $R \geq R_{0}$. In particular the term within the parentheses of the above inequality is positive and we can rewrite (3.49) in the form

$$
\left(\int_{\partial B_{R}} A(x) \frac{h_{+}(r)^{\delta}}{h_{-}(r)^{\delta}} h_{+}(r) \frac{\alpha(u)^{1+\delta}}{\beta(u)^{\delta}} e^{-\phi}\right)^{-\frac{1}{\delta}} \leq \frac{G^{\prime}(R)}{G(R)^{1+\frac{1}{\delta}}}
$$

on $\left[R_{0},+\infty\right)$. Hence, using (3.4),

$$
C_{0}^{-\frac{1}{\delta}}\left(\int_{\partial B_{R}} \frac{\alpha(u)^{1+\delta}}{\beta(u)^{\delta}} h_{+}(r) e^{-\phi}\right)^{-\frac{1}{\delta}} \leq \frac{G^{\prime}(R)}{G(R)^{1+\frac{1}{\delta}}} .
$$

Proceeding as in Lemma 1.1 of [24] we then obtain (3.41) with $C=\left(\delta^{\delta} C_{0}\right)^{-1}$. 
We are now ready to prove Theorem 3.4.

Proof of Theorem 3.4. Let $\zeta>0$ and set $\alpha(t)=e^{\zeta t}$, and $\beta(t)=\zeta e^{\zeta t}$. Hence (3.39), (3.40) are satisfied with $\kappa \equiv 0$. If $u$ is a solution of $L u \geq 0$ which is non constant and with $u^{*}=\sup _{M} u<+\infty$, applying (3.41) of Lemma 3.5 for $r>R \geq R_{0}$ we have

$$
\frac{1}{\zeta \int_{B_{R}} e^{\zeta u} \varphi(x,|\nabla u|)|\nabla u| h_{-}(r) e^{-\phi}} \geq C\left(\int_{R}^{r} \frac{1}{\left(\int_{\partial B_{t}} h_{+}(r) \frac{e^{u^{*}}}{\zeta} e^{-\phi}\right)^{\frac{1}{\delta}}}\right)^{\delta} .
$$

Letting $r \rightarrow+\infty$ and using (3.37) we obtain the desired contradiction.

\section{Killing graphs with prescribed mean curvature}

In this section, we deal with the equation of Killing graphs with prescribed mean curvature

$$
\operatorname{div}\left(\frac{\nabla u}{W}\right)-\left\langle\frac{\nabla \gamma}{2 \gamma}, \frac{\nabla u}{W}\right\rangle=n H,
$$

presented in Example 2.2.

Recall that $r=r(x)$ denotes the geodesic distance in $M$ from some fixed origin $o \in M$ and $B_{R}=B_{R}(o)$ stands for the geodesic ball centered at $o$ with radius $R$. We prove the following:

Theorem 4.1. Let $\bar{M}$ be a complete Riemannian manifold endowed with a complete Killing field $Y$ and let $M$ be an integral leaf of the Killing foliation. Assume that

$$
\sup _{M}|Y|<+\infty
$$

and

$$
\liminf _{R \rightarrow+\infty} \frac{\log \int_{B_{R}}|Y|}{R^{2-\varsigma-\mu}}=0,
$$

for some $\mu, \varsigma \in \mathbb{R}$, such that $\varsigma \geq 0$ and $2-\varsigma-\mu>0$.

Then there are no Killing graphs $\psi_{u}(x)=\Phi(x, u(x))$, for $x \in M$, lying between the graphs $\psi_{5, \pm \beta}(x)=\Phi\left(x, \pm \beta r(x)^{\varsigma}\right)$ outside a compact in $M$, for any $\beta>0$, and with mean curvature $H$ satisfying

$$
|H| \geq \frac{C}{(1+r(x))^{\mu}}
$$

for some $C>0$. 
Proof. We reason by contradiction and we let $\psi_{u}(x)$ be a Killing graph over $M$ satisfying the hypothesis of the theorem. Then $u$ is a solution of

$$
L u=\operatorname{div}_{\log \sqrt{\gamma}}\left(\frac{\nabla u}{W}\right)=n H \quad \text { on } M
$$

with $\gamma=|Y|^{-2}$. Since $H$ has constant sign, passing to $-u$ if necessary, we may assume that $H(x) \geq 0$ on $M$. To apply Theorem 3.2 we observe that $M$ is complete, we let $\phi=\log \sqrt{\gamma}=-\log |Y|$ and we choose $h$ to be the metric on $M$, so that $h_{-}$ and $h_{+}$are both identically equal to 1 . Define

$$
\varphi(x, t)=\frac{t}{\sqrt{\gamma(x)+t^{2}}} .
$$

Then $\varphi$ clearly satisfies i) and ii) in (3.3), while it satisfies iii) with the choices

$$
\delta=1 \quad \text { and } \quad A(x)=|Y(x)| .
$$

Thus, assumption (3.4) is guaranteed by (4.2). Since $\psi_{u}(x)$ lies between the graphs $\psi_{\varsigma,-\beta}(x)$ and $\psi_{\varsigma, \beta}(x)$, we have

$$
\widehat{u}=\limsup _{r(x) \rightarrow+\infty} \frac{u(x)}{r(x)^{\varsigma}} \leq \beta<+\infty .
$$

We now let $\tau=\mu+2(\varsigma-1)$ and observe that (3.6) is satisfied and (4.3) corresponds to (3.8) with $d_{0}=0$.

Because of assumption (4.4), we have

$$
(1+r(x))^{\mu} \operatorname{Lu}(x) \geq C>0
$$

on $M$. Next, we choose any $\xi$ such that $\Omega_{\xi}$ is non-empty to get, by applying Theorem 3.2, the desired contradiction, since

$$
\inf _{\Omega_{\xi}}(1+r(x))^{\mu} L u(x) \leq 0 .
$$

This concludes the proof of the theorem.

The reasoning in the above proof also shows the validity of the next:

Corollary 4.2. Let $\bar{M}$ be a complete Riemannian manifold endowed with a complete Killing field $Y$ and let $M$ be an integral leaf of the Killing foliation. Assume that

$$
\sup _{M}|Y|<+\infty
$$


and

$$
\liminf _{R \rightarrow+\infty} \frac{\log \int_{B_{R}}|Y|}{R^{2-\varsigma}}=0,
$$

for some $0 \leq \varsigma<2$.

Then any constant mean curvature Killing graph $\psi(x)=\Phi(x, u(x))$, for $x \in$ $M$, lying between the graphs $\psi_{5, \pm \beta}(x)=\Phi\left(x, \pm \beta r(x)^{\varsigma}\right)$ outside a compact in $M$, for some $\beta>0$, is minimal. In particular, if $\varsigma=0$, the sectional curvatures of $M$ satisfy $K_{M} \geq-K_{0}$ for some positive constant $K_{0}$ and $\operatorname{Ric}_{M} \geq 0$, then $\psi(M)$ is a leaf.

Proof. We only have to show the validity of the last statement. Since $u$ is bounded, by the main theorem in [12] we know that $|\nabla u|$ is bounded. Thus $u$ satisfies

$$
\operatorname{div}\left(\frac{\nabla u}{W}\right)-\left\langle\frac{\nabla \gamma}{2 \gamma}, \frac{\nabla u}{W}\right\rangle=0,
$$

where now the operator is uniformly elliptic because of the boundedness of $|\nabla u|$. Since $\operatorname{Ric}_{M} \geq 0, u$ is constant by [28, Theorem 7.4 ]. This finishes the proof.

Note that if we consider the density on $M$ given by $e^{-f}$ with $f=-\log |Y|=$ $\log \sqrt{\gamma}$ then (4.3) and (4.6) can be interpreted as conditions on the growth rate of the weighted volume of geodesic balls in $M$. In what follows we are going to show that this particular choice of weighted volume gives rise to a weighted isoperimetric ratio with relevant geometric implications.

For instance, it is well-known that entire graphs with constant mean curvature in the Euclidean space have to be minimal. I. Salavessa proved in [27] this result by an ingenious way of relating the mean curvature of the graph with the Cheeger's constant. We adapt her reasoning to the present situation.

Proposition 4.3. Given a function $u \in \mathcal{C}^{\infty}(M)$ suppose that the mean curvature $H$ of the graph of $u$ has constant sign, say $H>0$. Then

$$
\inf _{M} H \leq \frac{1}{n} \mathfrak{c}_{\log \sqrt{\gamma}},
$$

where

$$
\mathfrak{c}_{\log \sqrt{\gamma}}=\inf _{\Omega \subset M} \frac{\operatorname{vol}_{\log \sqrt{\gamma}}(\partial \Omega)}{\operatorname{vol}_{\log \sqrt{\gamma}}(\Omega)},
$$

with $\Omega$ running on the relatively compact domains with smooth boundary in $M$, is the weighted Cheeger's constant.

Proof. Since we can fix $H>0$, integrating (4.5) over a relatively compact domain $\Omega$ with smooth boundary yields

$$
\inf _{\Omega} n H \operatorname{vol}_{\log \sqrt{\gamma}}(\Omega) \leq \int_{\partial \Omega}\left\langle\frac{\nabla u}{W}, v\right\rangle|Y|,
$$


where

$$
\mathrm{vol}_{\log \sqrt{\gamma}}(\Omega)=\int_{\Omega}|Y|
$$

and $\nu$ is the unit normal along $\partial \Omega \subset M$. Then using Cauchy-Schwarz inequality we get

$$
\inf _{\Omega} H \leq \frac{1}{n} \frac{\operatorname{vol}_{\log \sqrt{\gamma}}(\partial \Omega)}{\operatorname{vol}_{\log \sqrt{\gamma}}(\Omega)} .
$$

This implies

$$
\inf _{M} H \leq \frac{1}{n} \mathfrak{c}_{\log \sqrt{\gamma}}
$$

with $\Omega$ running on the relatively compact domains with smooth boundary in $M$.

Of course (4.7) also holds if $H \geq 0$. Hence, turning back to Salavessa's result, in case $\bar{M}=\mathbb{R}^{n} \times \mathbb{R}$, the Killing vector field $Y$ is the coordinate vector field on the $\mathbb{R}$ factor and $H \geq 0$, the above yields

$$
\inf _{M} H=0 .
$$

This shows that there do not exist entire constant mean curvature graphs in $\bar{M}=$ $\mathbb{R}^{n} \times \mathbb{R}$ but the minimal ones. This can indeed be extended to the more general setting of Killing graphs. We have:

Proposition 4.4. Let $\operatorname{vol}_{\log \sqrt{\gamma}}\left(\partial B_{r}\right)=\int_{\partial B_{r}}|Y|$ and suppose that

$$
{ }^{{ }^{0 o l}} \log _{\sqrt{\gamma}}\left(\partial B_{r}\right) \leq C e^{a r^{\alpha}} \quad \text { on } \quad \mathbb{R}_{0}^{+}
$$

for some constants $C>0, a \geq 0, \alpha \in(0,1]$. Then

$$
\mathfrak{c}_{\log \sqrt{\gamma}} \begin{cases}=0 & \text { if } \alpha \in(0,1) \\ \leq a & \text { if } \alpha=1\end{cases}
$$

Proof. Proceeding as in Cheeger, [6], with the aid of the coarea formula, we obtain the (weighted) Cheeger's inequality

$$
\frac{\left(\mathfrak{c}_{\log \sqrt{\gamma}}\right)^{2}}{4} \leq \lambda_{1}^{\Delta_{\log \sqrt{\gamma}}}(M)
$$

where

$$
\lambda_{1}^{\Delta_{\log \sqrt{\gamma}}}(M)=\inf _{\substack{\varphi \in C_{C}^{\infty}(M), \varphi \neq 0}} \frac{\int_{M}|\nabla \varphi|^{2}|Y|}{\int_{M} \varphi^{2}|Y|}
$$


Then, adapting the proof of Theorem 7.8 of Bianchini, Mari, Rigoli, [5], one sees that

$$
\lambda_{1}^{\Delta_{\log \sqrt{\gamma}}}(M) \begin{cases}=0 & \text { if } \alpha \in(0,1) \\ \leq \frac{a^{2}}{4} & \text { if } \alpha=1 .\end{cases}
$$

Thus (4.9) follows from this latter and (4.10).

As an immediate consequence we have:

Corollary 4.5. Let $\bar{M}$ be a complete Riemannian manifold endowed with a complete Killing field $Y$ and let $M$ be an integral leaf of the Killing foliation. Suppose that

$$
\int_{\partial B_{r}}|Y| \leq C e^{a r^{\alpha}} \quad \text { on } \quad \mathbb{R}_{0}^{+}
$$

for some constants $c>0, a \geq 0, \alpha \in(0,1)$. Then any constant mean curvature Killing graph $\psi: M \rightarrow \bar{M}, \psi(x)=\Phi(x, u(x))$, for $x \in M$, is minimal.

We now comment about the sharpness of assumptions (4.3) and (4.6) in Theorem 4.1 and Corollary 4.2, respectively. Towards this aim, let us consider $\bar{M}=$ $\mathbb{H}^{n} \times \mathbb{R}$ and $Y$ the coordinate vector field on the $\mathbb{R}$ factor, as before. It is well-known that the Cheeger's constant of $\mathbb{H}^{n}$ is $n-1$. Therefore, we expect that the constant mean curvature with respect to an appropriate orientation of an entire graph is in the interval $\left[0, \frac{n-1}{n}\right]$.

However, in this case (4.6) is satisfied if and only if $\varsigma \in[0,1)$. Thus, from Corollary 4.2 , we deduce that every graph with constant mean curvature and lying between $\psi_{\varsigma, \pm \beta}(x)=\Phi\left(x, \pm \beta r(x)^{\varsigma}\right)$, for some $\beta>0$ and $\varsigma \in[0,1)$, is, in fact, minimal.

On the other hand, if $\varsigma \in[1,2)$ there do exist constant mean curvature graphs with $H \in\left(0, \frac{n-1}{n}\right]$ lying between the two graphs $\psi_{\varsigma, \pm \beta}$, for some $\beta>0$, outside a compact set in $\mathbb{H}^{n}$. To see this, let $\mathbb{H}^{n}$ be represented as the model space with metric of constant negative curvature -1 , which we realize in polar coordinates $(r, \theta) \in(0,+\infty) \times \mathbb{S}^{n-1}$ as

$$
\langle\cdot, \cdot\rangle=d r^{2}+\sinh ^{2}(r) d \theta^{2}
$$

$d \theta^{2}$ being the standard metric on $\mathbb{S}^{n-1}$.

Then, for any $H \in(0,(n-1) / n]$ the smooth function

$$
u(x)=\int_{0}^{r(x)} \frac{\sinh ^{1-n}(t) \int_{0}^{t} n H \sinh ^{n-1}(\tau) d \tau}{\sqrt{1-\sinh ^{2(1-n)}(t)\left(\int_{0}^{t} n H \sinh ^{n-1}(\tau) d \tau\right)^{2}}} d t
$$


defines an entire graph over $\mathbb{H}^{n}$ with constant mean curvature $H$. Furthermore,

$$
\lim _{r(x) \rightarrow+\infty} \frac{u(x)}{r(x)}=C_{H},
$$

where

$$
C_{H}= \begin{cases}\frac{n H}{\sqrt{(n-1)^{2}-n^{2} H^{2}}} & \text { if } 0<H<(n-1) / n \\ +\infty & \text { if } H=(n-1) / n .\end{cases}
$$

This means that for $H \in(0,(n-1) / n)$ the graph lies between the graphs

$$
\psi_{1, \pm 2 C_{H}}(x)=\left(x, \pm 2 C_{H} r(x)\right)
$$

in $\mathbb{H}^{n} \times \mathbb{R}$ outside a compact set of $\mathbb{H}^{n}$.

We further note that this example also shows that condition (4.6) cannot be relaxed to

$$
\liminf _{R \rightarrow+\infty} \frac{\log \int_{B_{R}}|Y|}{R^{2-\varsigma}} \in \mathbb{R}^{+} .
$$

Indeed, in the above case we have

$$
\liminf _{R \rightarrow+\infty} \frac{\log \int_{B_{R}}|Y|}{R^{2-\varsigma}}=\liminf _{R \rightarrow+\infty} \frac{\log \operatorname{vol} B_{R}}{R^{2-\varsigma}}= \begin{cases}+\infty & \text { if } \varsigma \in(1,2) \\ C(n-1) & \text { if } \varsigma=1\end{cases}
$$

for some $C>0$.

We conclude this section with the following observation: if $\varsigma=0$, i.e., $u$ is bounded, and $H(x)$ has constant sign, proceeding as in the proof of Theorem 4.1, we arrive to (4.5) with $u$ bounded above and $H(x) \geq 0$. Thus if (4.2) holds and

$$
\frac{1}{\int_{\partial B_{t}}|Y|} \notin L^{1}(+\infty)
$$

we can apply Theorem 3.4 to conclude that $(M,\langle\cdot, \cdot\rangle)$ is $L$-parabolic. As a consequence we have

Theorem 4.6. Let $\bar{M}$ be a complete Riemannian manifold endowed with a complete Killing field $Y$ and let $M$ be an integral leaf of the Killing foliation. Assume that

$$
\sup _{M}|Y|<+\infty
$$

and

$$
\frac{1}{\int_{\partial B_{t}}|Y|} \notin L^{1}(+\infty)
$$


Let $\psi_{u}(x)$ be a Killing graph with mean curvature of constant sign whose image lies in some slab of the form

$$
\Phi\left(M \times\left[s_{1}, s_{2}\right]\right)=\left\{\Phi(x, s): x \in M, \text { and } s_{1} \leq s \leq s_{2}\right\} .
$$

Then the graph is a leaf.

Remark 4.7. Note that condition (4.16) is much stronger than

$$
\liminf _{R \rightarrow+\infty} \frac{\log \int_{B_{R}}|Y|}{R^{2}}=0,
$$

which is nothing but (4.3) with $\varsigma=\mu=0$. For instance, if

$$
\int_{\partial B_{R}}|Y| \sim C e^{R^{2-\epsilon}} \text { as } R \rightarrow+\infty
$$

for some $C>0$ and $0<\epsilon<2$ then

$$
\log \int_{B_{R}}|Y| \sim C R^{2-\epsilon} \quad \text { as } \quad R \rightarrow+\infty .
$$

Hence, (4.17) is satisfied. But clearly, (4.16) is not met.

\subsection{The case of weighted Killing graphs}

If we fix $F(y, \eta)=|\eta|$ and choose a general weight $e^{-f}$ we obtain the setting of Killing graphs with prescribed weighted mean curvature. In this case, the EulerLagrange equation becomes

$$
\operatorname{div}_{f}\left(\frac{\nabla u}{W}\right)-\left\langle\frac{\nabla \gamma}{2 \gamma}, \frac{\nabla u}{W}\right\rangle=n H_{f},
$$

and we obtain the corresponding versions of Theorem 4.1 and Corollary 4.2, with the same proofs, and with the density $\phi$ in the operator $L$ defined in (3.1) now given by $\phi=f+\log \sqrt{\gamma}=f-\log |Y|$. Specifically, the corresponding versions are stated as follows:

Theorem 4.8. Let $\bar{M}$ be a complete Riemannian manifold endowed with a complete Killing field $Y$ and let $M$ be an integral leaf of the Killing foliation. Assume that

$$
\sup _{M}|Y|<+\infty
$$

and

$$
\liminf _{R \rightarrow+\infty} \frac{\log \int_{B_{R}}|Y| e^{-f}}{R^{2-\varsigma-\mu}}=0,
$$


for some $\mu, \varsigma \in \mathbb{R}$, such that $\varsigma \geq 0$ and $2-\varsigma-\mu>0$. Then there are no Killing graphs $\psi_{u}(x)=\Phi(x, u(x))$, for $x \in M$, lying between the graphs $\psi_{\varsigma, \pm \beta}(x)=$ $\Phi\left(x, \pm \beta r(x)^{5}\right)$ outside a compact in $M$, for any $\beta>0$, and with weighted mean curvature $H_{f}$ satisfying

$$
\left|H_{f}\right| \geq \frac{C}{(1+r(x))^{\mu}}
$$

for some $C>0$.

Corollary 4.9. Let $\bar{M}$ be a complete Riemannian manifold endowed with a complete Killing field $Y$ and let $M$ be an integral leaf of the Killing foliation. Assume that

$$
\sup _{M}|Y|<+\infty
$$

and

$$
\liminf _{R \rightarrow+\infty} \frac{\log \int_{B_{R}}|Y| e^{-f}}{R^{2-\varsigma}}=0
$$

for some $0 \leq \varsigma<2$.

Then any constant weighted mean curvature Killing graph $\psi(x)=\Phi(x, u(x))$, for $x \in M$, lying between the graphs $\psi_{\varsigma, \pm \beta}(x)=\Phi\left(x, \pm \beta r(x)^{\varsigma}\right)$ outside a compact in $M$, for some $\beta>0$, is minimal.

In order to emphasize the geometric nature of assumptions (4.22) and (4.24) on the growth rate of the weighted volume of geodesic balls in $M$, we digress about some examples which illustrate the sharpness of our results and the differences between the mean curvature case and its weighted counterpart.

For the sake of simplicity we suppose that the metric in $M$ is rotationally symmetric. More precisely, we suppose that $M=M_{\xi}$ is a model space with pole at $o$ and Gaussian coordinates $(r, \theta) \in \mathbb{R}^{+} \times \mathbb{S}^{n-1}$ in terms of which $\sigma$ is expressed by

$$
\sigma=\mathrm{d} r^{2}+\xi^{2}(r) \mathrm{d} \theta^{2}
$$

for some $\xi \in \mathcal{C}^{\infty}\left(\mathbb{R}_{0}^{+}\right)$, for $\xi>0$ in $\mathbb{R}^{+}$, satisfying $\xi^{\prime}(0)=1$, and $\xi^{(2 k)}(0)=0$, for each $k=0,1,2, \ldots$ and where $\mathrm{d} \theta^{2}$ denotes the canonical metric in $\mathbb{S}^{n-1}$.

We also assume that the norm of the Killing field does not depend on $\theta$. In this case, the ambient metric $\bar{g}$ of $\bar{M}$ is written in terms of cylindrical coordinates $(s, r, \theta)$ as

$$
\bar{g}=\varrho^{2}(r) \mathrm{d} s^{2}+\mathrm{d} r^{2}+\xi^{2}(r) \mathrm{d} \theta^{2},
$$

with $\varrho \in \mathbb{C}^{\infty}\left(\mathbb{R}_{0}^{+}\right), \varrho>0$ on $\mathbb{R}_{0}^{+}$. Note that $\bar{M}$ is a doubly-warped product with respect to warping functions of the coordinate $r$. This includes space forms as well as Riemannian products of space forms and the real line as particular examples of ambient manifolds. Here $Y=\partial / \partial s$, with $|Y|=\varrho(r)$, and we further assume that the density is a radial function, that is, $f=f(r)$. Let

$$
\chi(r)=\varrho(r) \xi^{n-1}(r) e^{-f(r)} .
$$


In this geometric setting, for a fixed $r_{0} \in \mathbb{R}^{+}$,

$$
h\left(r_{0}\right)=(n-1) \frac{\xi^{\prime}\left(r_{0}\right)}{\xi\left(r_{0}\right)}+\frac{\varrho^{\prime}\left(r_{0}\right)}{\varrho\left(r_{0}\right)}-f^{\prime}\left(r_{0}\right)=\frac{\chi^{\prime}\left(r_{0}\right)}{\chi\left(r_{0}\right)}
$$

is the weighted mean curvature of the Killing cylinder $r=r_{0}$ in $\bar{M}$ calculated with respect to the orientation $-\frac{\partial}{\partial r}$. Below we prove the existence of non-minimal entire graphs with constant weighted mean curvature.

Proposition 4.10. Let $\bar{M}$ with metric in cylindrical coordinates $(s, r, \theta)$ given by

$$
\varrho^{2}(r) d s^{2}+d r^{2}+\xi^{2}(r) d \theta^{2}
$$

as above. Assume that $\chi(r) \rightarrow+\infty$ as $r \rightarrow+\infty$ and

$$
\lim _{r \rightarrow 0^{+}} h(r)>0 \text { and } \quad \lim _{r \rightarrow+\infty} h(r)>0 .
$$

Then, there exist non-minimal entire rotationally symmetric graphs with weighted constant mean curvature $H_{f} \neq 0$, for every $n\left|H_{f}\right| \in\left(0, \inf _{\mathbb{R}^{+}} B(r)\right)$, where

$$
B(r)=\frac{\chi(r)}{\int_{0}^{r} \chi(t) d t} .
$$

Remark 4.11. As we will see in the proof, the assumptions on $h$ guarantee that $\inf _{\mathbb{R}^{+}} B(r)>0$, so that $\left(0, \inf _{\mathbb{R}^{+}} B(r)\right)$ is not empty.

Proof. Given a radial $\mathcal{C}^{2}$ function $u=u(r)$, equation (4.20) for constant weighted mean curvature $H_{f}$ becomes

$$
\operatorname{div}_{f+\log \sqrt{\gamma}}\left(\frac{u^{\prime}(r) \nabla r}{\sqrt{\gamma(r)+u^{\prime 2}(r)}}\right)=n H_{f} .
$$

Integrating on the geodesic ball $B_{r}$ with respect to the weighted measure $e^{-f-\log \sqrt{\gamma}} d M$ and using the divergence theorem we obtain

$$
\int_{\partial B_{r}} \frac{u^{\prime}}{\sqrt{\gamma+u^{\prime 2}}}|Y| e^{-f}=\int_{B_{r}} n H_{f}|Y| e^{-f} .
$$

Hence, using the co-area formula, we have

$$
\frac{u^{\prime}(r)}{\sqrt{\gamma+u^{\prime 2}(r)}} \varrho(r) \xi^{n-1}(r) e^{-f(r)}=n H_{f} \int_{0}^{r} \varrho(t) \xi^{n-1}(t) e^{-f(t)} d t
$$

and it follows that

$$
u(x)=u(r(x))=\int_{0}^{r(x)} \frac{\varrho^{-1}(t) \chi^{-1}(t) \int_{0}^{t} n H_{f} \chi(\tau) d \tau}{\sqrt{1-\chi^{-2}(t)\left(\int_{0}^{t} n H_{f} \chi(\tau) d \tau\right)^{2}}} d t
$$


provided

$$
n\left|H_{f}\right|<\inf _{\mathbb{R}^{+}} B(r) .
$$

Observe that, by de l'Hôpital's rule,

$$
\lim _{r \rightarrow 0^{+}} B(r)=\lim _{r \rightarrow 0^{+}} \frac{\chi^{\prime}(r)}{\chi(r)}>0 .
$$

On the other hand, since $\chi(r) \rightarrow+\infty$ as $r \rightarrow+\infty$, by the same rule we also have

$$
\lim _{r \rightarrow+\infty} B(r)=\lim _{r \rightarrow+\infty} \frac{\chi^{\prime}(r)}{\chi(r)}>0 .
$$

Therefore, $I=\left(0, \inf _{\mathbb{R}^{+}} B(r)\right) \neq \emptyset$. Hence (4.26) defines a radially symmetric Killing graph with constant non-zero weighted mean curvature $H_{f}$, for every

$$
n\left|H_{f}\right| \in I .
$$

This finishes the proof.

Observe that the example after Corollary 4.5 corresponds to the choices $\varrho \equiv 1$, $\xi(r)=\sinh (r)$ and $f \equiv 0$, for which one has

$$
\inf _{\mathbb{R}^{+}} B(r)=\inf _{\mathbb{R}^{+}} \frac{\sinh ^{n-1}(r)}{\int_{0}^{r} \sinh ^{n-1}(t) d t}=\lim _{r \rightarrow+\infty} \frac{\sinh ^{n-1}(r)}{\int_{0}^{r} \sinh ^{n-1}(t) d t}=n-1 .
$$

In contrast with Salavessa's result we have:

Proposition 4.12. There exist entire Killing graphs in Euclidean space with constant weighted mean curvature which are not minimal.

Proof. First note that $\xi(r)=r$ and $\varrho(r)=1$. Then fix any $\alpha \in \mathbb{R}$, and $\varepsilon>0$ and let

$$
e^{-f(r)}=\frac{e^{\varepsilon r}}{(1+r)^{\alpha}}
$$

A simple computation gives

$$
\inf _{\mathbb{R}^{+}} B(r)=\varepsilon .
$$

Defining $u(x)$ as in (4.26) for $n\left|H_{f}\right| \in(0, \varepsilon)$ we obtain a graph lying between the graphs $\psi_{1, \pm \beta}(x)= \pm \beta r(x)$ outside a compact set in $\mathbb{R}^{n}$.

We end this section with some comments about the necessity of (4.24). First observe that in the examples in the proof of Proposition 4.12 condition (4.24) is satisfied only for $\zeta \in[0,1)$. As for the asymptotic behavior of $u$ as $r \rightarrow \infty$, an easy computation shows that

$$
u(r) \sim C \log \chi(r) \quad \text { as } \quad r \rightarrow+\infty,
$$


for some constant $C>0$. For instance, consider again the case of $M=\mathbb{H}^{n}$ and choose $f(r)=-r^{\alpha}$ for some $\alpha>1$ and $r \gg 1$. Moreover, fix either $\varrho(r)=1$ or $\varrho(r)=\cosh (r)$. These choices correspond respectively to the Riemannian product $\mathbb{H}^{n} \times \mathbb{R}$ and to the hyperbolic space $\mathbb{H}^{n+1}=\mathbb{H}^{n} \times \cosh r \mathbb{R}$.

The above prescription gives us a graph $\psi_{u}$ for which

$$
u(r) \sim C r^{\alpha} \quad \text { as } \quad r \rightarrow+\infty
$$

for some constant $C>0$. In particular, $\psi_{u}(x)$ lies between the graphs $\psi_{5, \pm 2 C}(x)$ outside an appropriate compact set of $\mathbb{H}^{n}$ if and only if $\varsigma \geq \alpha$. On the other hand, (4.24) holds only for $\varsigma<2-\alpha$ which is excluded by our previous request. This shows the necessity of (4.24) also in this setting.

\subsection{An application of the parabolicity criterion}

In this subsection, we consider an intrisic weighted Laplacian operator defined on a Killing graph. We then apply Theorem 3.4 directly to such a graph with the Riemannian metric induced from the ambient space.

Theorem 4.13. Let $\bar{M}$ be a complete Riemannian manifold endowed with a complete Killing field $Y$ and let $M$ be an integral leaf of the Killing foliation. Assume that

$$
|Y|^{2} \in L^{1}(M, d M)
$$

Let $\psi_{u}(x)=\Phi(x, u(x))$, for $x \in M$, be a Killing graph on $M$ such that

$$
|\nabla u|=O\left(\frac{1}{|Y|}\right) \quad \text { as } x \rightarrow \infty
$$

If the mean curvature vector field points in the same (respectively, opposite) direction of $Y$ and $u$ is bounded from above (respectively, below), then $u$ is constant and the graph is a leaf of the foliation.

Proof. Let $\Sigma=\psi(M) \subset \bar{M}$ and denote by $\nabla^{\Sigma}$ and $\Delta_{\Sigma}$ the gradient and the Laplace operators on $\Sigma$ with respect to the induced metric. Then

$$
\nabla^{\Sigma} u=(\bar{\nabla} s)^{\top}=\gamma Y^{\top}
$$


where ${ }^{\top}$ denotes the tangential projection over $\Sigma$. Consider a local orthonormal frame $\left\{e_{1}, \ldots, e_{n}\right\}$ with respect to metric on $\Sigma$. Then

$$
\begin{aligned}
\Delta_{\Sigma} u & =\sum_{i=1}^{n}\left\langle\nabla_{e_{i}}^{\Sigma} \gamma Y^{\top}, e_{i}\right\rangle=\sum_{i=1}^{n}\left\langle\bar{\nabla}_{e_{i}} \gamma Y^{\top}, e_{i}\right\rangle \\
& =\gamma \sum_{i=1}^{n}\left\langle\bar{\nabla}_{e_{i}} Y^{\top}, e_{i}\right\rangle+\left\langle\bar{\nabla} \gamma, Y^{\top}\right\rangle \\
& =\gamma \sum_{i=1}^{n}\left\langle\bar{\nabla}_{e_{i}} Y, e_{i}\right\rangle-\gamma \sum_{i=1}^{n}\left\langle\bar{\nabla}_{e_{i}}\langle Y, N\rangle N, e_{i}\right\rangle+\left\langle\frac{\bar{\nabla} \gamma}{\gamma}, \gamma Y^{\top}\right\rangle \\
& =n H \gamma\langle Y, N\rangle+\left\langle\nabla^{\Sigma} u, \frac{\nabla^{\Sigma} \gamma}{\gamma}\right\rangle .
\end{aligned}
$$

That is,

$$
\Delta_{\Sigma, \log \gamma} u=n H \gamma\langle Y, N\rangle,
$$

where $\Delta_{\Sigma, \log \gamma}$ is the operator given by

$$
\Delta_{\Sigma, \log \gamma} u=\Delta_{\Sigma} u-\left\langle\nabla^{\Sigma} u, \nabla^{\Sigma} \log \gamma\right\rangle=e^{\phi} \operatorname{div}_{\Sigma}\left(e^{-\phi} \nabla^{\Sigma} u\right)
$$

with $\phi=\log \gamma$. Here $\operatorname{div}_{\Sigma}$ denotes the divergence on $\Sigma$ with respect to the induced metric.

We can now apply Theorem 3.4 to the operator $L=\Delta_{\Sigma, \log \gamma}$ on $\left(\Sigma,\langle\cdot, \cdot\rangle_{\Sigma}\right)$, with $\varphi(x, t)=t, \delta=1$ and $h=\langle\cdot, \cdot\rangle_{\Sigma}$, so that $h_{+}=1$, provided that condition (3.37) is satisfied. Note that in the present case condition (3.37) becomes

$$
\frac{1}{\int_{\partial B_{t}}|Y|^{2} d \Sigma} \notin L^{1}(+\infty),
$$

where $B_{t}$ is the geodesic ball of radius $t$ with respect to some fixed origin in the complete manifold $\left(\Sigma,\langle\cdot, \cdot\rangle_{\Sigma}\right)$.

According to [24, Proposition 1.3], condition (4.34) is satisfied if

$$
|Y|^{2} \in L^{1}(\Sigma, d \Sigma)
$$

Now observe that

$$
\langle\cdot, \cdot\rangle_{\Sigma}=\langle\cdot, \cdot\rangle_{M}+|Y|^{2} d u \otimes d u
$$

Hence, by (6.1) below,

$$
d \Sigma=\sqrt{1+|\nabla u|^{2}|Y|^{2}} d M
$$

On the other hand, by assumption (4.31) we have $|\nabla u|^{2}|Y|^{2} \leq C$ on $M$, for some constant $C>0$. It follows that

$$
d \Sigma \leq \sqrt{1+C} d M
$$


and assumption (4.30) implies (4.35). Therefore, Theorem 3.4 can be applied to the operator $\Delta_{\Sigma, \log \gamma}$. that

Finally, note that our assumptions on the mean curvature vector field guarantee

$$
(u-c) \Delta_{\Sigma, \log \gamma} u \leq 0
$$

for some constant $c$. Hence, by Theorem $3.4 u$ is constant. This finishes the proof of Theorem 4.13 .

\section{Killing graphs with prescribed anisotropic mean curvature}

Throughout this section, we consider the Lagrangian $F$ given by

$$
F(y, \eta)=a\left(\Theta\left(\frac{\eta}{|\eta|}\right)\right) \sqrt{g(\eta, \eta)}
$$

which is defined in Example 2.5. We are going to prove that the prescribed generalized mean curvature equation (2.10) may be written as (2.15). We have

$$
\begin{aligned}
\frac{\partial F}{\partial s}= & a\left(\Theta\left(\frac{\eta}{|\eta|}\right)\right) \frac{\partial}{\partial s} \sqrt{g(\eta, \eta)}+a^{\prime}\left(\Theta\left(\frac{\eta}{|\eta|}\right)\right) \frac{\partial}{\partial s} \Theta\left(\frac{\eta}{|\eta|}\right) \\
= & a\left(\Theta\left(\frac{\eta}{|\eta|}\right)\right) \frac{1}{2 \sqrt{g(\eta, \eta)}} \frac{\partial}{\partial s} g(\eta, \eta) \\
& +a^{\prime}\left(\Theta\left(\frac{\eta}{|\eta|}\right)\right)\left(£_{Y} \Theta\left(\frac{\eta}{|\eta|}\right)+\Theta\left(\left[\frac{\partial}{\partial s}, \frac{\eta}{|\eta|}\right]\right)\right) \\
= & a\left(\Theta\left(\frac{\eta}{|\eta|}\right)\right) \frac{1}{2 \sqrt{g(\eta, \eta)}}\left(£_{Y} g(\eta, \eta)+2 g\left(\left[\frac{\partial}{\partial s}, \eta\right], \eta\right)\right) \\
& +a^{\prime}\left(\Theta\left(\frac{\eta}{|\eta|}\right)\right)\left(£_{Y} \Theta\left(\frac{\eta}{|\eta|}\right)+\Theta\left(\left[\frac{\partial}{\partial s}, \frac{\eta}{|\eta|}\right]\right)\right) \\
= & a\left(\Theta\left(\frac{\eta}{|\eta|}\right)\right) \frac{1}{\sqrt{g(\eta, \eta)}} g\left(\left[\frac{\partial}{\partial s}, \eta\right], \eta\right)+a^{\prime}\left(\Theta\left(\frac{\eta}{|\eta|}\right)\right) \Theta\left(\frac{\partial}{\partial s},\left[\frac{\partial}{\partial s}, \frac{\eta}{|\eta|}\right]\right) .
\end{aligned}
$$

However, along a Killing graph

$$
\left[\frac{\partial}{\partial s}, \eta\right]=0
$$

and

$$
\frac{\partial}{\partial s}|\eta|=0
$$

from which we conclude that

$$
\left.\frac{\partial F}{\partial s}\right|_{\Sigma}=0
$$


To see the validity of (5.2), observe that

$$
\eta=\left.N\right|_{\Sigma}=-\frac{u^{i}}{W} \frac{\partial}{\partial x^{i}}+\frac{\gamma}{W} \frac{\partial}{\partial s},
$$

so that

$$
\begin{aligned}
{\left[\frac{\partial}{\partial s}, \eta\right]=} & -\frac{u^{i}}{W}\left[\frac{\partial}{\partial s}, \frac{\partial}{\partial x^{i}}\right]-\frac{\partial}{\partial s}\left(\frac{u^{i}}{W}\right) \frac{\partial}{\partial x^{i}} \\
& +\frac{\gamma}{W}\left[\frac{\partial}{\partial s}, \frac{\partial}{\partial s}\right]+\frac{\partial}{\partial s}\left(\frac{\gamma}{W}\right) \frac{\partial}{\partial s} \\
= & 0,
\end{aligned}
$$

because $\frac{u^{i}}{W}$ and $\frac{\gamma}{W}$ do not depend on $s$.

Hence, we may apply Theorem 2.1 in order to compute the generalized mean curvature of Killing graphs relatively to the Lagrangian $F$ defined in (5.1) that, in this case, corresponds to the anisotropic mean curvature of $\Sigma$, that we denote by $H_{F}$. We have

$$
\frac{\partial}{\partial \eta^{\alpha}} \sqrt{g(\eta, \eta)}=\frac{1}{\sqrt{g(\eta, \eta)}} g_{\alpha \beta} \eta^{\beta}
$$

and

$$
\frac{\partial}{\partial \eta^{\alpha}} \Theta\left(\frac{\eta}{|\eta|}\right)=\Theta_{\beta} \frac{\partial}{\partial \eta^{\alpha}} \frac{\eta^{\beta}}{|\eta|}=\frac{1}{|\eta|} \Theta_{\alpha}-\Theta\left(\frac{\eta}{|\eta|}\right) \frac{\eta_{\alpha}}{|\eta|^{2}},
$$

where the $\Theta_{\alpha}$ 's are the components of $\Theta$. It follows that

$$
\frac{\partial}{\partial \eta^{\alpha}} a(\Theta)=a^{\prime}(\Theta)\left(\frac{1}{|\eta|} \Theta_{\alpha}-\Theta \frac{\eta_{\alpha}}{|\eta|^{2}}\right)
$$

where to simplify the notation we have set $\Theta$ and $\Theta_{\alpha}$ for $\Theta\left(\frac{\eta}{|\eta|}\right)$ and $\Theta_{\alpha}\left(\frac{\eta}{|\eta|}\right)$, respectively. These formulas imply

$$
\frac{\partial F}{\partial \eta^{\alpha}}=a^{\prime}(\Theta) \Theta_{\alpha} \frac{\sqrt{g(\eta, \eta)}}{|\eta|}+a(\Theta) \frac{1}{\sqrt{g(\eta, \eta)}} g_{\alpha \beta} \eta^{\beta}-a^{\prime}(\Theta) \Theta \frac{\eta_{\alpha}}{|\eta|} \frac{\sqrt{g(\eta, \eta)}}{|\eta|}
$$

or equivalently

$$
\begin{aligned}
\frac{\partial F}{\partial \eta^{\alpha}}= & a^{\prime}(\Theta) \Theta_{\alpha} \frac{\sqrt{g(\eta, \eta)}}{|\eta|} \\
& +\left(a(\Theta) \frac{|\eta|}{\sqrt{g(\eta, \eta)}} g_{\alpha \beta}-a^{\prime}(\Theta) \Theta \frac{\sqrt{g(\eta, \eta)}}{|\eta|} \bar{g}_{\alpha \beta}\right) \frac{\eta^{\beta}}{|\eta|}
\end{aligned}
$$


In particular we have

$$
\frac{\partial F}{\partial \eta^{i}}=a^{\prime}(\Theta) \Theta_{i} \frac{\sqrt{g(\eta, \eta)}}{|\eta|}+\left(a(\Theta) \frac{|\eta|}{\sqrt{g(\eta, \eta)}} g_{i j}-a^{\prime}(\Theta) \Theta \frac{\sqrt{g(\eta, \eta)}}{|\eta|} \sigma_{i j}\right) \frac{\eta^{j}}{|\eta|} .
$$

However $\Theta_{i}=0$ and $g_{i j}=\sigma_{i j}$ for $1 \leq i, j \leq n$. Hence we obtain

$$
\sigma^{i j} \frac{\partial F}{\partial \eta^{i}}=\left(a(\Theta) \frac{|\eta|}{\sqrt{g(\eta, \eta)}}-a^{\prime}(\Theta) \Theta \frac{\sqrt{g(\eta, \eta)}}{|\eta|}\right) \frac{\eta^{j}}{|\eta|} .
$$

We observe that along the graph $\Sigma$ of $\psi: \Omega \rightarrow M$ setting $\eta=N$ it holds that

$$
\eta^{i}=-\frac{u^{i}}{W}, \quad|\eta|=1
$$

In the notations of Theorem 2.1 we then obtain

$$
X=\left(a^{\prime}(\Theta) \Theta \sqrt{g(N, N)}-a(\Theta) \frac{1}{\sqrt{g(N, N)}}\right) \frac{\nabla u}{W} .
$$

Therefore from (2.10)

$$
\begin{aligned}
n H_{F}= & \operatorname{div}\left(\left(a(\Theta) \frac{1}{\sqrt{g(N, N)}}-a^{\prime}(\Theta) \Theta \sqrt{g(N, N)}\right) \frac{\nabla u}{W}\right) \\
& -\left\langle\left(a(\Theta) \frac{1}{\sqrt{g(N, N)}}-a^{\prime}(\Theta) \Theta \sqrt{g(N, N)}\right) \frac{\nabla u}{W}, \frac{\nabla \gamma}{2 \gamma}\right\rangle,
\end{aligned}
$$

that is, equation (2.15) in Example 2.5. Note that

$$
\left.\Theta(N)\right|_{\Sigma}=\frac{1}{W}=\frac{1}{\sqrt{\gamma+|\nabla u|^{2}}}
$$

and

$$
\left.\sqrt{g(N, N)}\right|_{\Sigma}=\frac{1}{W} \sqrt{|\nabla u|^{2}+\lambda \gamma}
$$

imply

$$
\begin{aligned}
& \frac{1}{W}\left(a(\Theta) \frac{1}{\sqrt{g(N, N)}}-a^{\prime}(\Theta) \Theta \sqrt{g(N, N)}\right) \\
& =\frac{1}{\sqrt{\lambda \gamma+|\nabla u|^{2}}} a\left(\frac{1}{\sqrt{\gamma+|\nabla u|^{2}}}\right)-\frac{\sqrt{\lambda \gamma+|\nabla u|^{2}}}{\left(\gamma+|\nabla u|^{2}\right)^{\frac{3}{2}}} a^{\prime}\left(\frac{1}{\sqrt{\gamma+|\nabla u|^{2}}}\right) .
\end{aligned}
$$

In terms of the notation of Theorem 3.2 we choose $h$ the metric on $M$, so that $h_{-}$ and $h_{+}$are both identically equal to 1 , and we choose

$$
\varphi(x, t)=\frac{t}{\sqrt{\lambda \gamma+t^{2}}}\left(a\left(\frac{1}{\sqrt{\gamma+t^{2}}}\right)-\frac{\lambda \gamma+t^{2}}{\left(\gamma+t^{2}\right)^{\frac{3}{2}}} a^{\prime}\left(\frac{1}{\sqrt{\gamma+t^{2}}}\right)\right),
$$


so that

$$
\frac{1}{W}\left(a(\Theta) \frac{1}{\sqrt{g(N, N)}}-a^{\prime}(\Theta) \Theta \sqrt{g(N, N)}\right)=|\nabla u|^{-1} \varphi(x,|\nabla u|) .
$$

In the particular case when $a \equiv 1$ we have

$$
\varphi(x, t)=\frac{t}{\sqrt{\lambda \gamma+t^{2}}},
$$

while in the case $\lambda=1$ we obtain

$$
\varphi(x, t)=\frac{t}{\sqrt{\gamma+t^{2}}}\left(a\left(\frac{1}{\sqrt{\gamma+t^{2}}}\right)-\frac{1}{\sqrt{\gamma+t^{2}}} a^{\prime}\left(\frac{1}{\sqrt{\gamma+t^{2}}}\right)\right) .
$$

We are now able to apply Theorem 3.2 to obtain a non-existence result for graphs with prescribed anisotropic mean curvature.

Theorem 5.1. Let $\bar{M}$ be a complete Riemannian manifold endowed with a complete Killing field $Y$ and let $M$ be an integral leaf of the Killing foliation. Assume that

$$
\sup _{M}|Y|<+\infty
$$

and

$$
\liminf _{R \rightarrow+\infty} \frac{\log \int_{B_{R}}|Y|}{R^{2-\varsigma-\mu}}=0,
$$

for some $\mu, \varsigma \in \mathbb{R}, \varsigma \geq 0$, such that $2-\varsigma-\mu>0$.

Then there are no Killing graphs $\psi(x)=\Phi(x, u(x))$, for $x \in M$, lying between the graphs $\psi_{\varsigma, \pm \beta}(x)=\Phi\left(x, \pm \beta r(x)^{\varsigma}\right)$ outside a compact in $M$, for any $\beta>0$, and with anisotropic mean curvature $H_{F}$ satisfying

$$
\left|H_{F}\right| \geq \frac{C}{(1+r(x))^{\mu}}
$$

for some $C>0$, provided

$$
\Upsilon(w, z)>0 \quad \text { for every } \quad(w, z) \in \mathbb{R}^{+} \times \mathbb{R}^{+},
$$

and

$$
\Upsilon^{*}=\sup _{(w, z) \in \mathbb{R}^{+} \times \mathbb{R}^{+}} \Upsilon(w, z)<+\infty
$$

where

$$
\Upsilon(w, z)=a(z)-\left((\lambda-1) z^{2} w+1\right) a^{\prime}(z) z
$$

Remark 5.2. As we will see in the proof, condition (5.9) corresponds to the ellipticity condition ii) in Theorem 3.2. 
Proof. We reason by contradiction and we let $\psi(x)=\Phi(x, u(x))$ be a Killing graph over $M$ under the hypothesis of the theorem. Then $u$ satisfies

$$
L u=\operatorname{div}\left(|\nabla u|^{-1} \varphi(x,|\nabla u|) \nabla u\right)-|\nabla u|^{-1} \varphi(x,|\nabla u|)\left\langle\frac{\nabla \gamma}{2 \gamma}, \nabla u\right\rangle=n H_{F}
$$

with $\gamma=|Y|^{-2}$. We choose $f=\log \sqrt{\gamma}=-\log |Y|$ and $\varphi(x, t)$ given by (5.5). Now, the proof goes along the same lines of the proof of Theorem 4.1, with the choices

$$
\delta=1 \quad \text { and } \quad A(x)=\frac{\Upsilon^{*}}{\sqrt{\lambda}}|Y(x)| .
$$

Clearly $\varphi$ satisfies i) in Theorem 3.2. Condition ii) is guaranteed because of (5.9), observing that

$$
a\left(\frac{1}{\sqrt{\gamma+t^{2}}}\right)-\frac{\lambda \gamma+t^{2}}{\left(\gamma+t^{2}\right)^{\frac{3}{2}}} a^{\prime}\left(\frac{1}{\sqrt{\gamma+t^{2}}}\right)=\Upsilon(w, z)
$$

with

$$
w=\gamma \quad \text { and } \quad z=\frac{1}{\sqrt{\gamma+t^{2}}} .
$$

Finally, iii) is granted because of (5.10) and the choices of $\delta$ and $A(x)$.

On the other hand, assumption (3.4) is satisfied because of (5.6). As in Theorem 4.1, (3.6) is satisfied with $\tau=\mu+2(\varsigma-1)$, while (5.7) corresponds to (3.8) with $d_{0}=0$. The rest of the proof is the same as in Theorem 4.1.

Similarly to the previous section, we have

Corollary 5.3. Let $\bar{M}$ be a complete Riemannian manifold endowed with a complete Killing field $Y$ and let $M$ be an integral leaf of the Killing foliation. Assume that

$$
\sup _{M}|Y|<+\infty
$$

and

$$
\liminf _{R \rightarrow+\infty} \frac{\log \int_{B_{R}}|Y|}{R^{2-\varsigma}}=0
$$

for some $0 \leq \varsigma<2$.

Then, under assumptions (5.9) and (5.10), any Killing graph $\psi(x)=$ $\Phi(x, u(x))$, for $x \in M$, with constant anisotropic mean curvature lying between the graphs $\psi_{\varsigma, \pm \beta}(x)=\Phi\left(x, \pm \beta r(x)^{\varsigma}\right)$ outside a compact in $M$, for some $\beta>0$, is minimal. 


\subsection{An anisotropic Weingarten map}

In this subsection, we interpret the anisotropic mean curvature as the trace of a tensor which plays the role of the Weingarten map in the anisotropic setting. In particular, this shows that our definition of generalized mean curvature extends the usual one for immersed hypersurfaces. To accomplish this, we must assume that $F$ is horizontally constant, i.e., the total derivative $D F$ of $F$ in $T \bar{M}$ has no horizontal component.

In order to give a precise meaning to this statement, we fix some terminology and basic facts on the geometry of the tangent bundle $T \bar{M}$.

The canonical projection $\pi: T \bar{M} \rightarrow \bar{M}, \pi(y, \eta)=y$, determines the vertical distribution

$$
(y, \eta) \in T \bar{M} \mapsto \mathcal{V}_{(y, \eta)}=\left.\operatorname{ker} \pi_{*}\right|_{(y, \eta)} \subset T_{(y, \eta)} T \bar{M},
$$

which is integrable with integral leaves given by the fibers $\pi^{-1}(y)=T_{y} \bar{M}$. The Riemannian connection $\bar{\nabla}$ in $\bar{M}$ determines a horizontal distribution

$$
(y, \eta) \in \bar{M} \mapsto \mathcal{H}_{(y, \eta)}=\operatorname{ker} K_{(y, \eta)} \subset T_{(y, \eta)} T \bar{M},
$$

where the map $K: T T \bar{M} \rightarrow T \bar{M}$ is defined by

$$
K_{(y, \eta)}\left(U_{*} \zeta\right)=\bar{\nabla}_{\zeta} U, \quad \text { for } \zeta \in T_{y} \bar{M},
$$

where $U \in \Gamma(T \bar{M})$ is a vector field in $\bar{M}$ with $U(y)=\eta$. It turns out that horizontal and vertical subspaces are orthogonal with respect to the Sasaki metric $\langle\cdot, \cdot\rangle_{T} \bar{M}$ in $T \bar{M}$ defined by

$$
\langle\mathcal{Y}, \mathcal{W}\rangle_{T \bar{M}}=\left\langle\pi_{*} \mathcal{Y}, \pi_{*} \mathcal{W}\right\rangle+\langle K \mathcal{Y}, K \mathcal{W}\rangle, \quad \mathcal{Y}, \mathcal{W} \in \Gamma(T T \bar{M}) .
$$

Orthogonal projections onto vertical and horizontal subspaces are respectively denoted by $\pi_{*}^{\mathrm{v}}$ and $\pi_{*}^{\mathrm{h}}$. In terms of the local coordinates defined earlier in Section 2, the vertical and horizontal lifts of tangent vectors in $\bar{M}$ are given by

$$
\begin{aligned}
& \left.\left(\frac{\partial}{\partial y^{k}}\right)^{\mathrm{v}}\right|_{(y, \eta)}=\left.\frac{\partial}{\partial \eta^{k}}\right|_{(y, \eta)}, \\
& \left.\left(\frac{\partial}{\partial y^{k}}\right)^{\mathrm{h}}\right|_{(y, \eta)}=\left.\frac{\partial}{\partial y^{k}}\right|_{(y, \eta)}-\left.\Gamma_{k l}^{r}(y) \eta^{l} \frac{\partial}{\partial \eta^{r}}\right|_{(y, \eta)},
\end{aligned}
$$

for $1 \leq k \leq n+1$, where $\Gamma_{k l}^{r}$ are the Christoffel symbols of $\bar{\nabla}$ calculated with respect to the local chart $y^{1}, \ldots, y^{n+1}$. Vertical and horizontal lifts preserve the metric in the sense that

$$
\left\langle U^{\mathrm{h}}, V^{\mathrm{h}}\right\rangle_{T \bar{M}}=\left\langle U^{\mathrm{v}}, V^{\mathrm{v}}\right\rangle_{T \bar{M}}=\langle U, V\rangle,
$$


for any vector fields $U, V \in \Gamma(T \bar{M})$. This implies that the fibers $\pi^{-1}(y)$, for $y \in \bar{M}$, are totally geodesic. Thus, the coordinates $\left(\eta^{k}\right)_{k=1}^{n+1}$ defined above consist of a global normal coordinate system for each fiber. More precisely, it holds that the Riemannian connection $D$ in $T \bar{M}$ determined by the Sasaki metric satisfies

$$
D_{U^{\vee}} V^{\mathrm{v}}=0
$$

for any $U, V \in \Gamma(T \bar{M})$.

Definition 5.4. Given a cross-section $\chi(x)=(\psi(x), N(\psi(x)))$, for $x \in \Sigma$, of the fiber bundle $\psi^{*} T \bar{M}$, we define a covariant tensor $\mathcal{A}_{F} \in \Gamma\left(T^{*} \Sigma \otimes T^{*} \Sigma\right)$ by

$$
\mathcal{A}_{F}(U, V)=\left\langle D_{\left(\psi_{*} U\right)^{\vee}} D F,\left(\psi_{*} V\right)^{\mathrm{v}}\right\rangle_{T \bar{M}} \circ \chi,
$$

for any vector fields $U, V \in \Gamma(T \Sigma)$. We also define the tensor $\mathcal{A}_{F}^{\sharp} \in \Gamma\left(T \Sigma \otimes T^{*} \Sigma\right)$ by

$$
\left\langle\mathcal{A}_{F}^{\sharp} U, V\right\rangle=\mathcal{A}_{F}(U, V), \quad \text { for } \quad U, V \in \Gamma(T \Sigma) \text {. }
$$

We observe that the tensor $\mathcal{A}_{F}^{\sharp}$ is well-defined taking tangent vector fields over tangent vector fields, due to the degree one homogeneity of $F$. We also remark that the tensors $\mathcal{A}_{F}$ and $\mathcal{A}_{F}^{\sharp}$ are symmetric.

In local coordinates we have

$$
\mathcal{A}_{F}\left(\psi_{*} \frac{\partial}{\partial x^{i}}, \psi_{*} \frac{\partial}{\partial x^{j}}\right)=\left.\frac{\partial y^{k}}{\partial x^{i}} \frac{\partial y^{l}}{\partial x^{j}} \frac{\partial^{2} F}{\partial \eta^{k} \partial \eta^{l}}\right|_{\chi(x)} .
$$

Definition 5.5. The tensor $A_{F} \in \Gamma\left(T \Sigma \otimes T^{*} \Sigma\right)$ defined by

$$
\left\langle A_{F} U, V\right\rangle=\left\langle\mathcal{A}_{F}^{\sharp} A U, V\right\rangle=\mathcal{A}_{F}(A U, V), \quad \text { for } \quad U, V \in \Gamma(T \Sigma),
$$

is the anisotropic Weingarten map, where $A \in \Gamma\left(T \Sigma \otimes T^{*} \Sigma\right)$ is the usual Weingarten map of $\psi$ corresponding to the normal $N$.

Definition 5.6. A parametric Lagrangian $F: T \bar{M} \backslash\{0\} \rightarrow \mathbb{R}$ is said to be horizontally constant if $\pi_{*}^{\mathrm{h}} D F=0$.

Note that, in Euclidean space, this amounts to imposing that $F(y, \eta)=F(\eta)$.

Definition 5.6 is particularly meaningful since it enables us to recover the anisotropic mean curvature defined, in variational terms in Theorem 2.1, as the trace of the tensor $A_{F}$. Indeed, we have the following result whose proof can be found in [22].

We follow the notations of Section 2.

Theorem 5.7. If the parametric Lagrangian $F: T \bar{M}-\{0\} \rightarrow \mathbb{R}$ is horizontally constant, then the anisotropic Weingarten map is given by

$$
\psi_{*} A_{F}=-\bar{\nabla} X
$$

with $X$ as in (2.9). In this case $n H_{F}=\operatorname{tr} A_{F}$. 
In the particular case of a Riemannian product $\bar{M}=M \times \mathbb{R}$ for which $Y=\frac{\partial}{\partial s}$, the Lagrangian

$$
F(y, \eta)=a(\Theta)|\eta|
$$

with $a \in \mathcal{C}^{2}(\mathbb{R})$ and $\Theta=\left\langle Y, \frac{\eta}{|\eta|}\right\rangle$, is horizontally constant. Note that (5.25) is a special case of (5.1) with $\lambda=1$. In this case we easily verify that

$$
\pi_{*}^{\vee} D F=a(\Theta) \frac{\eta}{|\eta|}+a^{\prime}(\Theta)\left(Y-\Theta \frac{\eta}{|\eta|}\right) .
$$

Here, ' denotes derivative with respect to the real variable $\Theta$. Furthermore, the parallelism of $Y$ implies that $F$ is horizontally constant. Indeed, given any vector field $U \in \Gamma(T \bar{M})$, we have

$$
\left\langle D F, U^{\mathrm{h}}\right\rangle_{T \bar{M}}=a^{\prime}(\Theta)\left\langle\bar{\nabla}_{U} Y, \eta\right\rangle=0 .
$$

Hence, in this setting the anisotropic mean curvature is the trace of the anisotropic Weingarten map.

\section{Appendix: proof of Theorem 2.1}

Let $\Omega$ be a relatively compact domain in $M$ with smooth boundary. Since $F$ is a degree-one homogeneous function with respect to the second variable, we have

$$
\begin{aligned}
\mathcal{F}_{f}[\psi] & =\int_{\Omega} F(\psi, N) e^{-f} \psi^{*} d \bar{M} \\
& =\int F\left(\psi, \frac{\psi_{*} \frac{\partial}{\partial x^{1}} \wedge \ldots \wedge \psi_{*} \frac{\partial}{\partial x^{n}}}{\left|\psi_{*} \frac{\partial}{\partial x^{1}} \wedge \ldots \wedge \psi_{*} \frac{\partial}{\partial x^{n}}\right|}\right) e^{-f} \sqrt{\operatorname{det} g_{i j}} d x \\
& =\int F\left(\psi, \psi_{*} \frac{\partial}{\partial x^{1}} \wedge \ldots \wedge \psi_{*} \frac{\partial}{\partial x^{n}}\right) e^{-f} d x
\end{aligned}
$$

where we have used the fact that

$$
\sqrt{\operatorname{det} g_{i j}}=\sqrt{\operatorname{det}\left\langle\psi_{*} \frac{\partial}{\partial x^{i}} \psi_{*} \frac{\partial}{\partial x^{j}}\right\rangle}=\left|\psi_{*} \frac{\partial}{\partial x^{1}} \wedge \ldots \wedge \psi_{*} \frac{\partial}{\partial x^{n}}\right| .
$$

Observing that

$$
g_{i j}, \quad \text { for } \quad 1 \leq i, j \leq n,
$$

are the components of the metric induced on $\Omega$ by the immersion $\psi$, we compute

$$
\begin{aligned}
& \psi_{*} \frac{\partial}{\partial x^{1}} \wedge \ldots \wedge \psi_{*} \frac{\partial}{\partial x^{n}}=\left(\frac{\partial}{\partial x^{1}}+u_{1} \frac{\partial}{\partial s}\right) \wedge \ldots \wedge\left(\frac{\partial}{\partial x^{n}}+u_{n} \frac{\partial}{\partial s}\right) \\
= & \frac{\partial}{\partial x^{1}} \wedge \ldots \wedge \frac{\partial}{\partial x^{n}}+\sum_{i=1}^{n} u_{i} \frac{\partial}{\partial x^{1}} \wedge \ldots \wedge \frac{\partial}{\partial x^{i-1}} \wedge \frac{\partial}{\partial s} \wedge \frac{\partial}{\partial x^{i+1}} \wedge \ldots \wedge \frac{\partial}{\partial x^{n}} .
\end{aligned}
$$


Hence,

$$
\begin{aligned}
\psi_{*} & \frac{\partial}{\partial x^{1}} \wedge \ldots \wedge \psi_{*} \frac{\partial}{\partial x^{n}}=\left|\frac{\partial}{\partial x^{1}} \wedge \ldots \wedge \frac{\partial}{\partial x^{n}}\right| \frac{\frac{\partial}{\partial s}}{\left|\frac{\partial}{\partial s}\right|} \\
& -\left|\frac{\partial}{\partial s}\right|\left|\frac{\partial}{\partial x^{1}} \wedge \ldots \wedge \frac{\partial}{\partial x^{n}}\right| \sum_{i=1}^{n} \sigma^{i j} u_{j} \frac{\partial}{\partial x^{i}} \\
= & \left|\frac{\partial}{\partial x^{1}} \wedge \ldots \wedge \frac{\partial}{\partial x^{n}}\right|\left|\frac{\partial}{\partial s}\right|\left(\gamma \frac{\partial}{\partial s}-\Phi_{*} \nabla u\right)=\frac{1}{\sqrt{\gamma}}\left|\frac{\partial}{\partial x^{1}} \wedge \ldots \wedge \frac{\partial}{\partial x^{n}}\right| W N \\
= & \frac{1}{\sqrt{\gamma}} \sqrt{\operatorname{det} \sigma_{i j}} W N=: G W N,
\end{aligned}
$$

where we have set

$$
G=\frac{1}{\sqrt{\gamma}} \sqrt{\operatorname{det} \sigma_{i j}}
$$

and

$$
W=\left|\gamma \frac{\partial}{\partial s}-\Phi_{*} \nabla u\right|=\sqrt{\gamma+|\nabla u|^{2}} .
$$

It follows that

$$
\sqrt{\operatorname{det} g_{i j}}=\frac{1}{\sqrt{\gamma}} \sqrt{\gamma+|\nabla u|^{2}} \sqrt{\operatorname{det} \sigma_{i j}} .
$$

We also conclude that the coordinates of the point $\left(\psi, \psi_{*} \frac{\partial}{\partial x^{1}} \wedge \ldots \wedge \psi_{*} \frac{\partial}{\partial x^{n}}\right)$ in $T \bar{M}$ are given by

$$
y^{0}=u, y^{1}=x^{1}, \ldots, y^{n}=x^{n}
$$

and

$$
\eta^{0}=\gamma G, \quad \eta^{i}=-G u^{i}
$$

Next, we consider a variation of the immersion $\psi: \Omega \rightarrow \bar{M}$ defined by

$$
\psi_{t}(x)=\Phi(x, u(x)+t v(x)), \quad \text { for } \quad x \in \Omega, t \in \mathbb{R},
$$

where $v: \Omega \rightarrow \mathbb{R}$ is a compactly supported $\mathcal{C}^{2}$ function. Differentiating, one obtains

$$
\begin{aligned}
\frac{d e^{-f} F}{d t} & =e^{-f} \frac{\partial F}{\partial s} \frac{d}{d t}(u+t v)+e^{-f} \frac{\partial F}{\partial x^{i}} \frac{d x^{i}}{d t}+e^{-f} \frac{\partial F}{\partial \eta^{0}} \frac{d}{d t}(\gamma G)-e^{-f} \frac{\partial F}{\partial \eta^{i}} \frac{d}{d t}\left(G u^{i}\right) \\
& =e^{-f} \frac{\partial F}{\partial s} v-e^{-f} \frac{\partial F}{\partial \eta^{i}} G v^{i},
\end{aligned}
$$

where we used the fact that $f$ does not depend on $s$.

Noting that

$$
\frac{G_{i}}{G}=-\frac{\gamma_{i}}{2 \gamma}+\frac{(\sqrt{\operatorname{det} \sigma})_{i}}{\sqrt{\operatorname{det} \sigma}}
$$


we obtain

$$
\begin{aligned}
e^{-f} \frac{\partial F}{\partial \eta^{i}} G v^{i}= & e^{-f} \frac{\partial F}{\partial \eta^{i}} G \sigma^{i j} v_{j}=\frac{\partial}{\partial x^{j}}\left(e^{-f} \sigma^{i j} \frac{\partial F}{\partial \eta^{i}} G v\right)-\frac{\partial}{\partial x^{j}}\left(e^{-f} \sigma^{i j} \frac{\partial F}{\partial \eta^{i}} G\right) v \\
= & \frac{\partial}{\partial x^{j}}\left(e^{-f} \sigma^{i j} \frac{\partial F}{\partial \eta^{i}} G v\right)-\frac{\partial}{\partial x^{j}}\left(\sigma^{i j} \frac{\partial F}{\partial \eta^{i}}\right) e^{-f} G v \\
& -\sigma^{i j} \frac{\partial F}{\partial \eta^{i}}\left(-f_{j}-\frac{\gamma_{j}}{2 \gamma}+\frac{(\sqrt{\operatorname{det} \sigma})_{j}}{\sqrt{\operatorname{det} \sigma}}\right) e^{-f} G v \\
= & \frac{\partial}{\partial x^{j}}\left(e^{-f} \sigma^{i j} \frac{\partial F}{\partial \eta^{i}} G v\right)-\left(\frac{\partial}{\partial x^{j}}\left(\sigma^{i j} \frac{\partial F}{\partial \eta^{i}}\right)+\sigma^{i j} \frac{\partial F}{\partial \eta^{i}} \frac{(\sqrt{\operatorname{det} \sigma})_{j}}{\sqrt{\operatorname{det} \sigma}}\right) e^{-f} G v \\
& +\sigma^{i j} \frac{\partial F}{\partial \eta^{i}}\left(f_{j}+\frac{\gamma_{j}}{2 \gamma}\right) e^{-f} G v .
\end{aligned}
$$

However, by equation (3.4.9) in [30] (see also in [10, Chapter 1]), we have

$$
\frac{(\sqrt{\operatorname{det} \sigma})_{j}}{\sqrt{\operatorname{det} \sigma}}=\Gamma_{j k}^{k},
$$

and thus,

$$
\begin{aligned}
e^{-f} \frac{\partial F}{\partial \eta^{i}} G v^{i}= & \frac{\partial}{\partial x^{j}}\left(e^{-f} \sigma^{i j} \frac{\partial F}{\partial \eta^{i}} G v\right) \\
& -\left(\frac{\partial}{\partial x^{j}}\left(\sigma^{i j} \frac{\partial F}{\partial \eta^{i}}\right)+\sigma^{i j} \frac{\partial F}{\partial \eta^{i}} \Gamma_{j k}^{k}-\sigma^{i j} \frac{\partial F}{\partial \eta^{i}} f_{j}\right) e^{-f} G v \\
& +\sigma^{i j} \frac{\partial F}{\partial \eta^{i}} \frac{\gamma_{j}}{2 \gamma} e^{-f} G v .
\end{aligned}
$$

Hence, we finally have

$$
\begin{aligned}
\frac{d e^{-f} F}{d t}= & e^{-f} \frac{\partial F}{\partial s} v-\frac{\partial}{\partial x^{j}}\left(e^{-f} \sigma^{i j} \frac{\partial F}{\partial \eta^{i}} G v\right) \\
& +\left(\sigma^{i j} \frac{\partial F}{\partial \eta^{i}}\right)_{; j} e^{-f} G v-\sigma^{i j} \frac{\partial F}{\partial \eta^{i}} f_{j} e^{-f} G v-\sigma^{i j} \frac{\partial F}{\partial \eta^{i}} \frac{\gamma_{j}}{2 \gamma} e^{-f} G v .
\end{aligned}
$$

Letting

$$
X=\left.\sigma^{i j} \frac{\partial F}{\partial \eta^{i}}\right|_{\left(\psi, \psi_{*} \frac{\partial}{\partial x^{1}} \wedge \ldots \wedge \psi_{*} \frac{\partial}{\partial x^{n}}\right)} \frac{\partial}{\partial x^{j}}
$$

one has

$$
\begin{aligned}
\frac{d e^{-f} F}{d t} & =e^{-f} \frac{\partial F}{\partial s} v-\frac{\partial}{\partial x^{j}}\left(e^{-f} X^{j} G v\right)+\left(X_{; j}^{j}-X^{j} f_{j}\right) e^{-f} G v-\sigma^{i j} \frac{\partial F}{\partial \eta^{i}} \frac{\gamma_{j}}{2 \gamma} e^{-f} G v \\
& =e^{-f} \frac{\partial F}{\partial s} v-\frac{\partial}{\partial x^{j}}\left(e^{-f} X^{j} G v\right)+\operatorname{div}_{f} X e^{-f} G v-\left\langle X, \frac{\nabla \gamma}{2 \gamma}\right\rangle e^{-f} G v .
\end{aligned}
$$


Now we use the assumption $\left.£_{Y} F\right|_{\Sigma}=0$, that is,

$$
\left.\frac{\partial F}{\partial s}\right|_{\Sigma}=0 .
$$

Thus, since $v$ is compactly supported in $\Omega$, we get

$$
\begin{aligned}
\left.\frac{d}{d t}\right|_{t=0} \mathcal{F}_{f}\left[\psi_{t}\right] & =\int-\frac{\partial}{\partial x^{j}}\left(e^{-f} X^{j} G v\right)+\operatorname{div}_{f} X e^{-f} G v-\left\langle X, \frac{\nabla \gamma}{2 \gamma}\right\rangle e^{-f} G v \\
& =\int\left(\operatorname{div}_{f} X-\left\langle X, \frac{\nabla \gamma}{2 \gamma}\right\rangle\right) e^{-f} G v \\
& =\int_{\Omega} \frac{1}{\sqrt{\gamma}}\left(\operatorname{div}_{f} X-\left\langle X, \frac{\nabla \gamma}{2 \gamma}\right\rangle\right) v e^{-f} \sqrt{\operatorname{det} \sigma} d x .
\end{aligned}
$$

We conclude that the Euler-Lagrange equation for unconstrained critical points of $\mathcal{F}_{f}$ is given by

$$
\operatorname{div}_{f} X-\left\langle X, \frac{\nabla \gamma}{2 \gamma}\right\rangle=0
$$

where

$$
X=\left.\sigma^{i j} \frac{\partial F}{\partial \eta^{i}}\right|_{\left(\psi, \psi_{*} \frac{\partial}{\partial x^{1}} \wedge \ldots \wedge \psi_{*} \frac{\partial}{\partial x^{n}}\right)} \frac{\partial}{\partial x^{j}} .
$$

But each derivative $\frac{\partial F}{\partial \eta^{i}}$ is homogeneous of degree zero, so that

$$
X=\left.\sigma^{i j} \frac{\partial F}{\partial \eta^{i}}\right|_{(\psi, N)} \frac{\partial}{\partial x^{j}} .
$$

This proves the validity of equation (2.8). To deal with the second part of the theorem we have to consider the modified functional

$$
\mathcal{F}_{f}[\psi]+\mathcal{V}_{f}[\psi]
$$

where

$$
\mathcal{V}_{f}[\psi]=\int_{\Omega} n H \frac{u}{\sqrt{\gamma}} e^{-f} d M
$$

is the volume enclosed by the graph with respect to the measure $e^{-f} d \bar{M}$. It is then a standard procedure to derive the validity of (2.10) (see, for instance, [3] and [4]). This concludes the proof of the theorem. 


\section{References}

[1] G. Albanese, L. J. AlíAs and M. Rigoli, A general form of the weak maximum principle and some applications, Rev. Mat. Iberoam. 29 (2013), 1437-1476.

[2] B. ANDREWS, Volume-preserving anisotropic mean curvature flow, Indiana Univ. Math. J. 50 (2001), 783-827.

[3] J. L. BARBOSA and M. DO CARMO, Stability of hypersurfaces with constant mean curvature, Math. Z. 185 (1984), 339-353.

[4] J. L. Barbos A, M. Do Carmo and J.-H. Eschenburg, Stability of hypersurfaces of constant mean curvature in Riemannian manifolds, Math. Z. 197 (1988), 123-138.

[5] B. BIANCHINI, L. MARI and M. RigOLI, On some aspects of oscillation theory and geometry, Mem. Amer. Math. Soc. 225, (2013).

[6] J. CHEEgER, A lower bound for the smallest eigenvalue of the Laplacian, In: "Problems in Analysis" (Papers dedicated to Salomon Bochner, 1969), Princeton Univ. Press, Princeton, N. J., 1970, 195-199.

[7] U. Clarenz, Enclosure theorems for extremals of elliptic parametric functionals, Calc. Var. Partial Differential Equations 15 (2002), 313-324.

[8] U. Clarenz and H. VON DER Mosel, On surfaces of prescribed F-mean curvature, Pacific J. Math. 213 (2004), 15-36.

[9] T. H. Colding and W. MinicozzI II, Estimates for parametric elliptic integrands, Int. Math. Res. Not. 6 (2002), 291-297.

[10] B. Chow, P. LU and L. Ni, "Hamilton's Ricci Flow", Graduate Studies in Mathematics, Vol. 77, American Mathematical Society, Providence, RI; Science Press, New York, 2006.

[11] M. DAJCZER, P. HinojosA and J. H. DE LIRA, Killing graphs with prescribed mean curvature, Calc. Var. Partial Differential Equations 33 (2008), 231-248.

[12] M. DAJCZER and J. H. DE LIRA, Entire bounded constant mean curvature Killing graphs, J. Math. Pures Appl. 103 (2015), 219-227.

[13] K. DECKELNICK and G. DZIUK, Discrete anisotropic curvature flow of graphs, M2AN Math. Modelling Numer. Anal. (RAIRO) 33 (1999), 1203-1222.

[14] G. DZIUK, Discrete anisotropic curve shortening flow, SIAM J. Numer. Anal. 36 (1999), $1808-1830$.

[15] Y. GigA, "Surface Evolution Equations - a Level set Approach", Monographs in Mathematics, Vol. 99, Birkhäuser Verlag, Basel, 2006.

[16] Y. HE and H. LI, Stability of hypersurfaces with constant $(r+1)$-th anisotropic mean curvature, Illinois J. Math. 52 (2008), 1301-1314.

[17] S. Hildebrandt and H. VON Der Mosel, Plateau's problem for parametric double integrals. I. Existence and regularity in the interior, Comm. Pure Appl. Math. 56 (2003), 926-955.

[18] S. Hildebrandt and H. VON DeR Mosel, Plateau's problem for parametric double integrals. II. Regularity at the boundary, J. Reine Angew. Math. 56 (2003), 207-233.

[19] M. KoIso and B. PALMER, Geometry and stability of surfaces with constant anisotropic mean curvature, Indiana Univ. Math. J. 54 (2005), 1817-1852.

[20] M. KoISO and B. PALMER, Stability of anisotropic capillary surfaces between two parallel planes, Calc. Var. Partial Differential Equations 25 (2006), 275-298.

[21] N. KOREVAAR, An easy proof of the interior gradient bound for solutions to the prescribed mean curvature equation, In: "Nonlinear Functional Analysis and its Applications", Part 2 (Berkeley, Calif., 1983), Proc. Sympos. Pure Math., Vol. 45, Part 2, Amer. Math. Soc., Providence, RI, 1986, 81-89.

[22] J. H. DE LIRA and M. MELO, Hypersurfaces with constant anisotropic mean curvature in Riemannian manifolds, Calc. Var. Partial Differential Equations 50 (2014), 335-364.

[23] S. Pigola, M. Rigoli and A. Setti, Maximum principles on Riemannian manifolds and applications, Mem. Amer. Math. Soc. 174 (2005). 
[24] M. Rigoli and A. SETTI, Liouville-type theorems for $\phi$-subharmonic functions, Rev. Mat. Iberoam. 17 (2001), 471-520.

[25] M. Rigoli, M. S ALVATORI and M. Vignati, Some remarks on the weak maximum principle, Rev. Mat. Iberoam. 21 (2005), 459-481.

[26] H. ROSENBERG, F. SCHUlZE and J. SPRUCK, The half-space property and entire positive minimal graphs in $M \times \mathbb{R}$. J. Differential Geom. 95 (2013), 321-336.

[27] I. M. SalaVessa, Graphs with parallel mean curvature, Proc. Amer. Math. Soc. 107 (1989), 449-458.

[28] L. SALOFF-Coste, Uniformly elliptic operators on Riemannian manifolds, J. Differential Geom. 36 (1992), 417-450.

[29] J. E. TAYLOR, Crystalline variational problems, Bull. Amer. Math. Soc. 84 (1978), 568588.

[30] R. M. WALD, “General Relativity”, University of Chicago Press, Chicago, IL, 1984.

Departamento de Matematicas

Universidad de Murcia

E30100 - Espinardo

Murcia, Spain

ljalias@um.es

Departamento de Matematica

Universidade Federal do Ceará

Bloco 914 - Campus do Pici

60455-760 - Fortaleza - Ceará, Brazil

jorge.lira@mat.ufc.br

Dipartimento di Matematica

Università degli Studi di Milano

Via Saldini, 50

20133 - Milano, Italia

marco.rigoli@unimi.it 